Document downloaded from:

http://hdl.handle.net/10251/98369

This paper must be cited as:

Mario Montagud; Fernando Boronat; Roig, B.; Sapena Piera, A. (2017). How to Perform AMP? Cubic Adjustments for Improving the QoE. Computer Communications. 103:61-73. doi:10.1016/j.comcom.2017.01.017

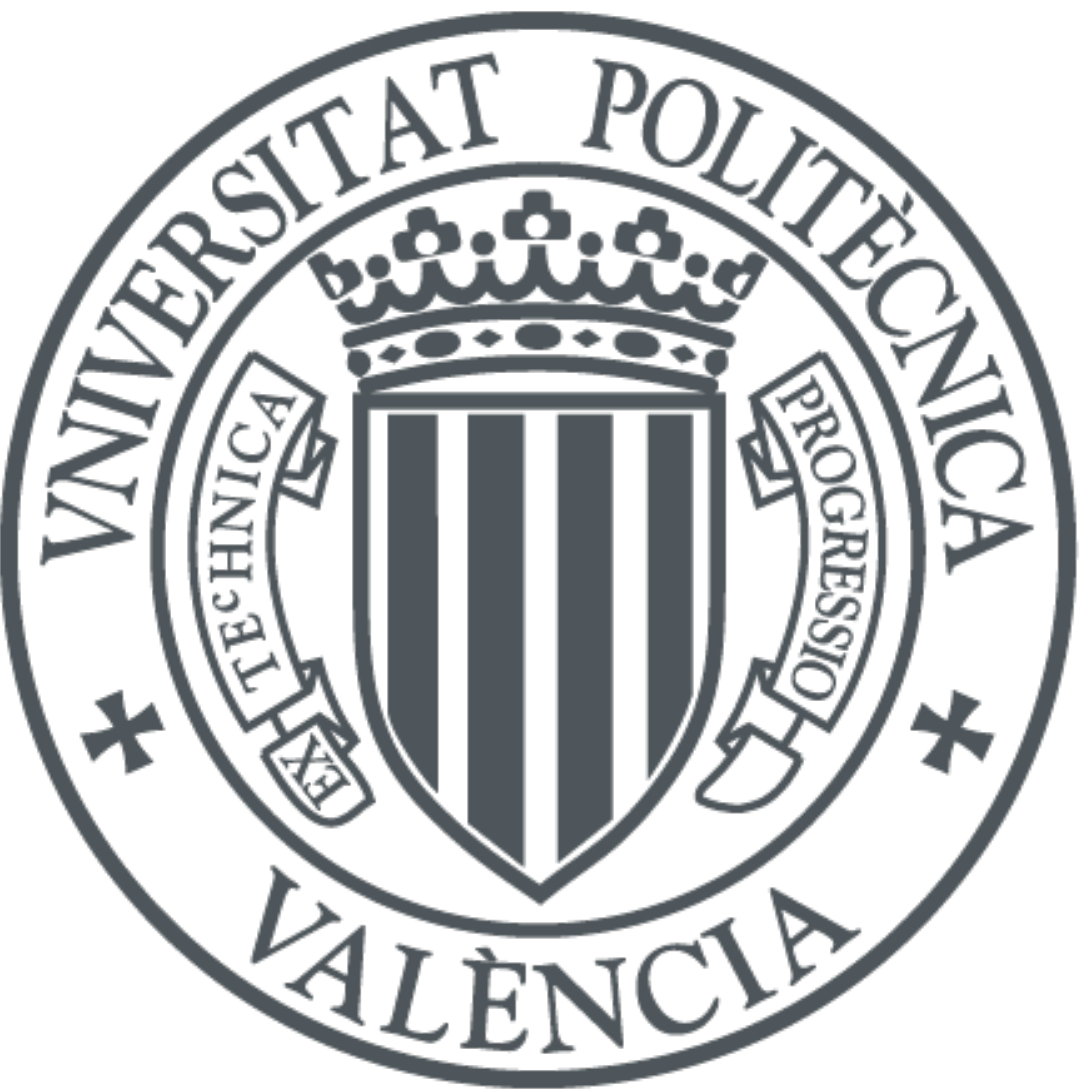

The final publication is available at

https://doi.org/10.1016/j.comcom.2017.01.017

Copyright Elsevier

Additional Information 


\section{How to Perform AMP? Cubic Adjustments for Improving the QoE}

Mario Montagud, Fernando Boronat, Bernardino Roig, and Almanzor Sapena Campus de Gandia - Universitat Politècnica de València (UPV) Grao de Gandia, Valencia (Spain)

mamontor@upv.es; fboronat@dcom.upv.es; broig@mat.upv.es; alsapie@mat.upv.es

Abstract - Adaptive Media Playout (AMP) consists of smoothly and dynamically adjusting the media playout rate to recover from undesired (e.g., buffer overflow/underflow or out-of-sync) situations. The existing AMP solutions are mainly characterized by two main aspects. The first one is their goal (e.g., keeping the buffers' occupancy into safe ranges or enabling media synchronization). The second one is the criteria that determine the need for triggering the playout adjustments (e.g., buffer fullness or asynchrony levels). This paper instead focuses on a third key aspect, which has not been sufficiently investigated yet: the specific adjustment strategy to be performed. In particular, we propose a novel AMP strategy, called Cubic AMP, which is based on employing a cubic interpolation method to adjust a deviated playout point to a given reference. On the one hand, mathematical analysis and graphical examples show that our proposal provides superior performance than other existing linear and quadratic AMP strategies in terms of the smoothness of the playout curve, while significantly outperforming the quadratic AMP strategy regarding the duration of the adjustment period and without increasing the computational complexity. It has also been proved and discussed that higher-order polynomial interpolation methods are less convenient than cubic ones. On the other hand, the results of subjective tests confirm that our proposal provides better Quality of Experience (QoE) than the other existing AMP strategies.

Keywords - Adaptive Media Playout; Cubic Interpolation; Inter-Destination Media Synchronization; Media Synchronization; QoE 


\section{Graphical Abstract + Highlights:}

\section{$\underline{\text { Adaptive Media Playout (AMP) Strategies }}$}

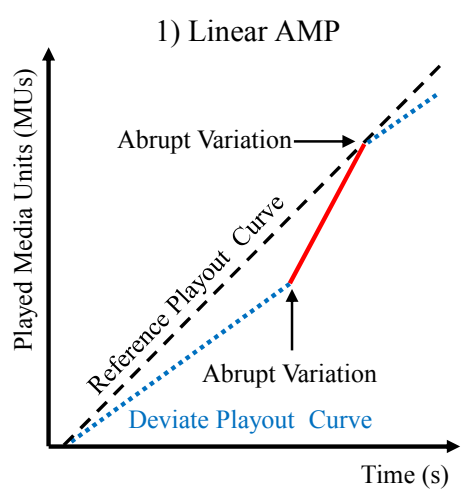

2.2) Quadratic AMP

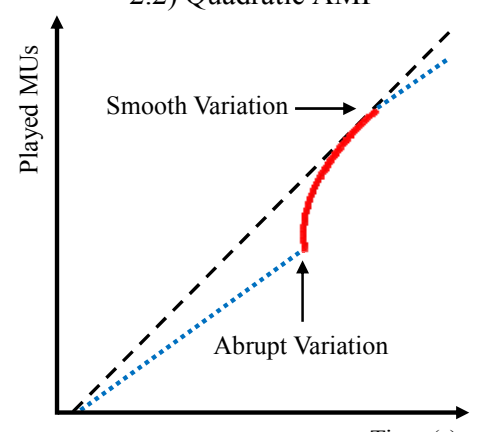

Time (s)
2.1) Quadratic AMP

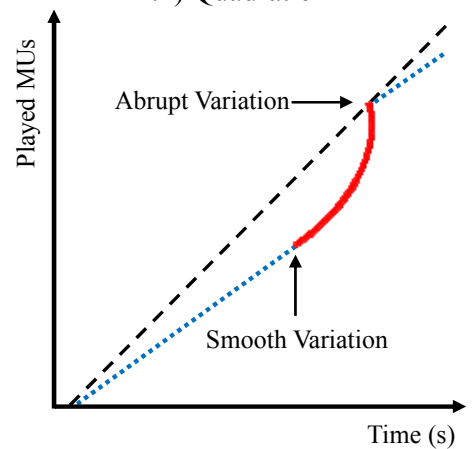

3) Cubic AMP

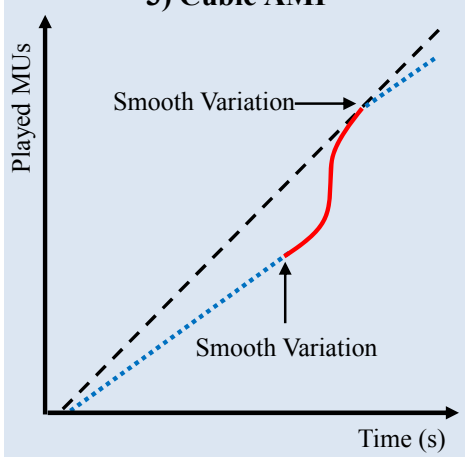

\section{$\underline{\text { Cubic AMP }}$}

- Smooth playout adjustment techniques are much more convenient than aggressive ones (i.e., skips \& pauses).

- Up to date, Linear and Quadratic Adaptive Media Playout (AMP) strategies have been devised.

- We propose a Cubic AMP strategy to adjust a deviated playout point to a given reference.

- Cubic AMP is the most suitable and simple solution, because:

- It guarantees the smoothness of the playout curve, avoiding bends.

- It reduces the duration of the adjustment period (only outperformed by Linear AMP).

- It keeps the computational complexity low.

- Cubic AMP provides better Quality of Experience (QoE) than other existing Linear and Quadratic AMP strategies.

- Slowing down the playout rate is more critical than fasting it up.

- Cubic AMP can be used for different goals (e.g., buffer underflow/overflow avoidance or media synchronization).

\section{List of Acronyms:}

$\begin{array}{ll}\text { AMP } & \text { Adaptive Media Playout } \\ \text { CBR } & \text { Constant Bit Rate } \\ \text { CoD } & \text { Content on Demand } \\ \text { CPU } & \text { Central Processing Unit } \\ \text { FEC } & \text { Forward Error Correction } \\ \text { IPTV } & \text { Internet Protocol Television } \\ \text { IDMS } & \text { Inter-Destination Multimedia Synchronization } \\ \text { ITU } & \text { International Telecommunication Union } \\ \text { MOS } & \text { Mean Opinion Score } \\ \text { MU } & \text { Media Unit } \\ \text { PSNR } & \text { Peak Signal-to-Noise Ratio } \\ \text { QoE } & \text { Quality of Experience } \\ \text { QoS } & \text { Quality of Service } \\ \text { RMS } & \text { Root Mean Square } \\ \text { VBR } & \text { Variable Bit Rate } \\ \text { VoD } & \text { Video on Demand }\end{array}$




\section{Introduction.}

Nowadays, we are witnessing a boom of media streaming services over Internet, and this is expected to grow further in the near future. Audio/video conferencing, Content on Demand (CoD) and Internet Protocol Television (IPTV) are just a few examples of these services.

However, the delivery of time-sensitive media content over packet-switched (IP) networks still faces many challenges. Several factors, such as variable bandwidth, jitter, and packet loss, among others, can prevent from successfully providing low-latency, interactive and synchronized media services $[1,2]$.

Typically, playout buffering strategies are employed at the client side to overcome the impact of the above factors, contributing to optimize the system performance in terms of: i) end-to-end delays; ii) intra-media synchronization (sync, hereafter), which is the maintenance of the original temporal relationships between the Media Units (MUs) within each media element (e.g., audio, video...); iii) inter-media sync, which is the preservation of the temporal dependences between the involved media elements (e.g., lip-sync); and iv) Inter-Destination Media Sync (or IDMS), which is the compensation of delay differences between different receivers $[1,2]$. All the above issues have an impact on the Quality of Service (QoS) and on the user's perceived Quality of Experience (QoE), especially when interactivity with the media content, and/or between multiple users within the context of common media content consumption, is pursued (e.g., in Social TV).

With enough bandwidth availability and network stability, the usage of proper buffering strategies would ideally allow preserving/reconstructing the original timing of the incoming media contents (i.e., $\mu_{\text {ideal }} \approx \theta \mathrm{MU} / \mathrm{s}$, being $\theta$ the generation rate at the server side and $\mu$ the playout rate at the client side, as shown in the black curve in Figure 1). However, the existence of additional factors, such as network congestion/limitations, Central Processing Unit (CPU) overload/limitations and clock imperfections, will have an impact on the media playout continuity and/or synchronicity. For example, the existence of playout rate imperfections [1, 2], such as skews $(\varphi)$ - deviation trends - and drifts $(\omega(t))$ - non-linear fluctuations - (i.e., $\mu_{\text {real }}(t) \approx \theta \cdot(1+\varphi+\omega(t))$ $\mathrm{MU} / \mathrm{s}$ ), will result in deviated (e.g., lagged or advanced) playout processes, as illustrated in Figure 1. Due to this, the adopted buffering strategies must be also coordinated with playout adjustment techniques to recover from undesired situations, such as buffer underflow/overflow or out-of-sync situations.

Two main approaches can be adopted for adjusting the playout processes: aggressive and smooth (see Figure 1). Aggressive adjustment techniques consist of simply skipping (i.e., discarding) or pausing MUs (also, inserting or duplicating MUs), as can be seen in the dotted purple lines in Figure 1.a. However, they can cause (long-term) playout disruptions or discontinuities (a.k.a. stallings), with a consequent degradation of the QoE, as shown in [3] and in [4]. Smooth adjustment techniques are commonly known as Adaptive Media Playout (AMP) and are based on varying (i.e., speeding up or slowing down) the media playout rate within tolerable 
ranges, as can be seen in the dashed red lines in Figure 1.b. Previous studies have shown that smooth adjustment techniques are more convenient than aggressive ones, in terms of QoS, such as in providing better media sync performance $[1,2,3]$ and in reducing delays $[5,6]$, and of the perceived QoE [3, 4].

Given these benefits, many AMP solutions have been proposed in the past (briefly described and classified in Section 2). In general, the goal of the existing AMP solutions is to adapt a deviated (lagged or advanced) playout point in order to reach a reference (target), being this reference: $i$ ) a target buffer fullness level in intra-media sync (e.g., to recover the buffer occupancy into safe ranges in case of network or end-systems congestion); ii) the playout point of a reference element in inter-media sync (e.g., the base stream in scalable video coding, the audio stream in audiovisual communications...); or iii) the playout point of a reference receiver in IDMS (e.g., possible strategies can be found in [2]).

However, independently of the particular goal of each AMP solution (i.e., why to perform the playout adjustments), and of criteria or conditions that are employed to determine the need for triggering the playout adjustments in each of them (i.e., when to perform the playout adjustments), an additional key issue is the selection of the specific interpolation method to reach the reference (i.e., how to perform the playout adjustments). Regarding this last aspect, most of the existing AMP solutions are based on performing linear (i.e., constant) playout adjustments until the reference point (i.e., the target) is reached. However, the work in [5] states that a quadratic (i.e., parabolic) adjustment function is better suited than a linear adjustment function for improving the QoE, as it allows reducing the distortion of the playout curve (i.e., it provides a more uniform playout, by keeping the inter-presentation times of successive MUs as uniform as possible).

a) Agressive Adjustments (Skips \& Pauses)

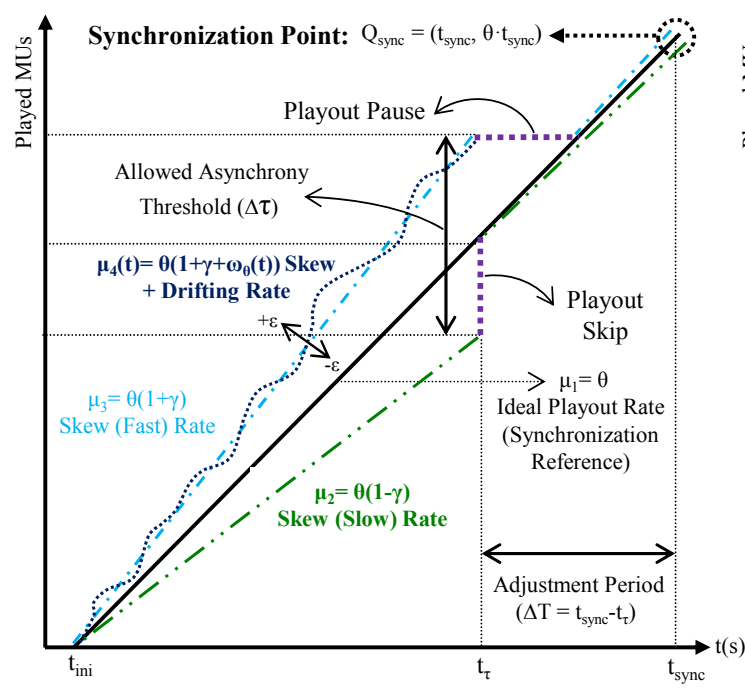

b) Smooth Adjustments (Linear AMP)

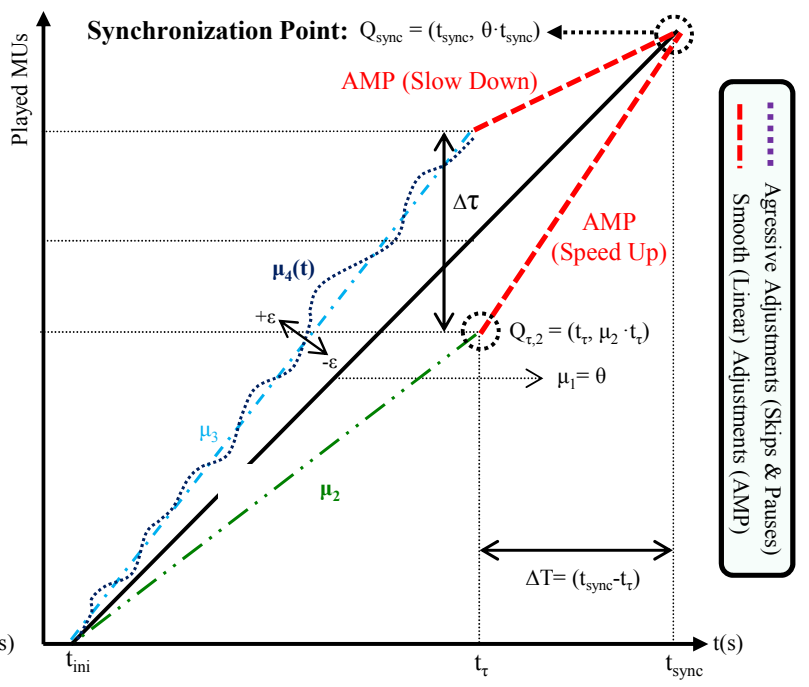

Figure 1. Playout Rate Deviations and Adjustment Techniques. 
Due to the fact that the smoothness of the playout curve is a key contributor to a satisfying QoE, the goal of this paper is to investigate and demonstrate if other strategies can provide better performance in terms of minimizing abrupt variations in the playout curve, but also in reducing the duration of the adjustment period ${ }^{1}$, while keeping the computational complexity low. Consequently, we propose a novel AMP strategy, called Cubic AMP, which is based on employing a cubic interpolation method to adjust a deviated playout point to a given reference, with the aim of minimizing abrupt changes in the media playout curve, thus improving its smoothness. Mathematical analysis and graphical representations show that the proposed Cubic AMP strategy meets the targeted requirements, and that the use of higher-order polynomial interpolation methods does not provide any benefit compared to the use of cubic interpolation methods. Most importantly, the results of subjective tests confirm that Cubic AMP provides better QoE than the other existing (linear and quadratic) AMP strategies.

The rest of this paper is organized as follows. Section 2 reviews the state-of-the-art regarding the existence of AMP solutions. In Section 3, the proposed Cubic AMP strategy is presented. In that section, in order to better understand and demonstrate the benefits provided by our proposal, the AMP process is firstly introduced and modeled, and then the expressions for the interpolation curves and for the duration of the adjustment periods when using the existing (linear and quadratic) AMP strategies are compared to the new ones obtained for Cubic AMP. It is also discussed that higher-order polynomial interpolation methods are less convenient than cubic ones. Section 4 provides the results of the objective and subjective evaluations that have been conducted, showing the benefits of using Cubic AMP. Finally, Section 5 outlines the conclusions and suggests some ideas for future work

\section{Related Work}

Many AMP solutions have been proposed in previous works to provide better QoS and/or QoE in a variety of multimedia systems and services. In this section, these solutions are reviewed, by highlighting the goal they are trying to achieve (i.e., the problem to be solved), the criteria that determine the need for triggering the playout adjustments, the involved media types (audio, video ...), the application scenario and the employed evaluation methodology, among other relevant aspects. Most interestingly (within the context of this work), the type of performed playout adjustments in each of these AMP solutions is identified: linear (i.e., Linear AMP, from now on) or quadratic (i.e., Quadratic AMP, from now on). A taxonomy of these existing AMP solutions, based on the previously mentioned aspects, is provided in Table 1.

\footnotetext{
${ }^{1}$ To the best of authors' knowledge, this factor has not been considered up to date when designing and evaluating AMP solutions, even though previous works have shown its significant influence on the QoE [3].
} 
Table 1. Taxonomy of Adaptive Media Playout (AMP) Solutions

\begin{tabular}{|c|c|c|c|c|c|c|c|}
\hline Ref & Goal (Why?) & $\begin{array}{c}\text { Criteria / } \\
\text { Metric } \\
\text { (When?) }\end{array}$ & $\begin{array}{l}\text { Media } \\
\text { Types }\end{array}$ & Adjustments & $\begin{array}{c}\text { Evaluation } \\
\text { Scenario }\end{array}$ & $\begin{array}{c}\text { Evaluation } \\
\text { Methodology }\end{array}$ & Video Clip \\
\hline$[3]$ & Inter-media Sync & $\begin{array}{l}\text { Asynchrony } \\
\text { between } \\
\text { Media Types }\end{array}$ & $\begin{array}{l}\text { Audio } \\
+ \\
\text { Video }\end{array}$ & $\begin{array}{l}\text { Fast Up / } \\
\text { Slow Down } \\
\text { (Linear AMP) }\end{array}$ & $\begin{array}{c}\text { Video } \\
\text { Conferencing }\end{array}$ & $\begin{array}{c}\text { QoS } \\
\text { (Simulation) }+ \\
\text { QoE tests }\end{array}$ & $\begin{array}{l}\text { Traces of } \\
\text { Audio/Video } \\
\text { Conference } \\
\text { clips }\end{array}$ \\
\hline$[6]$ & $\begin{array}{l}\text { Intra-media Sync } \\
\text { (Avoidance of } \\
\text { Buffer Underflow } \\
/ \text { Overflow } \\
\text { Situations) } \\
\quad+ \\
\text { Reduction of the } \\
\text { Initial Playout } \\
\text { Delay }\end{array}$ & $\begin{array}{l}\text { Lower } \\
\text { thresholds for } \\
\text { the Buffer } \\
\text { occupancy }\end{array}$ & $\begin{array}{l}\text { Audio } \\
+ \\
\text { Video }\end{array}$ & $\begin{array}{l}\text { Fast Up / } \\
\text { Slow Down } \\
\text { (Linear } \\
\text { AMP) }\end{array}$ & $\begin{array}{l}\text { VoD in } \\
\text { Wireless } \\
\text { Secenarios }\end{array}$ & $\begin{array}{c}\text { QoS } \\
\text { (Simulation) }\end{array}$ & $\begin{array}{l}\text { Constant Bit } \\
\text { Rate (CBR) } \\
\text { Video Traces }\end{array}$ \\
\hline$[8]$ & $\begin{array}{l}\text { Intra-media Sync } \\
\text { (Avoidance of } \\
\text { Buffer Underflow } \\
\text { Situations) }\end{array}$ & $\begin{array}{l}\text { Content- } \\
\text { aware }\end{array}$ & Video & $\begin{array}{l}\text { Slow Down } \\
\text { (Linear } \\
\text { AMP) }\end{array}$ & VoD & $\begin{array}{c}\text { QoS } \\
\text { (Simulation) }+ \\
\text { QoE tests }\end{array}$ & $\begin{array}{l}\text { Video clips } \\
\text { with different } \\
\text { motion intensity } \\
\text { (no audio) }\end{array}$ \\
\hline [9] & $\begin{array}{l}\text { Intra-media Sync } \\
\text { (Avoidance of } \\
\text { Buffer Underflow } \\
\text { / Overflow } \\
\text { Situations) }\end{array}$ & $\begin{array}{l}\text { Buffer } \\
\text { occupancy } \\
\text { variation }\end{array}$ & Video & $\begin{array}{l}\text { Fast Up / } \\
\text { Slow Down } \\
\text { (Linear } \\
\text { AMP) }\end{array}$ & VoD & $\begin{array}{c}\text { QoS } \\
\text { (Simulation) }\end{array}$ & $\begin{array}{l}\text { Simulated } \\
\text { traffic pattern } \\
\text { for a video } \\
\text { stream ( } 30 \mathrm{fps} \text {, } \\
\text { no details about } \\
\text { packet sizes). }\end{array}$ \\
\hline $\begin{array}{l}{[1],} \\
{[2]}\end{array}$ & IDMS & $\begin{array}{l}\text { Asynchrony } \\
\text { between } \\
\text { receivers }\end{array}$ & Video & $\begin{array}{l}\text { Fast Up / } \\
\text { Slow Down } \\
\text { (Linear } \\
\text { AMP) }\end{array}$ & $\begin{array}{c}\text { Video } \\
\text { Streaming }\end{array}$ & $\begin{array}{c}\text { QoS } \\
\text { (Simulation) }\end{array}$ & $\begin{array}{l}\text { VBR } \\
\text { traces }\end{array}$ \\
\hline$[10]$ & IDMS & $\begin{array}{l}\text { Content- } \\
\text { aware }^{\mathrm{a}} \\
\text { selection of } \\
\text { video } \\
\text { sequences } \\
\end{array}$ & $\begin{array}{l}\text { Audio } \\
+ \\
\text { Video }\end{array}$ & $\begin{array}{l}\text { Fast Up / } \\
\text { Slow Down } \\
\text { (Linear } \\
\text { AMP) }\end{array}$ & $\begin{array}{c}\text { Web-based } \\
\text { VoD }\end{array}$ & $\begin{array}{c}\text { QoE } \\
\text { (Crowsourcing) }\end{array}$ & Sintel \\
\hline [11] & IDMS & $\begin{array}{l}\text { Randomly } \\
\text { selected } \\
\text { content } \\
\text { sections }\end{array}$ & $\begin{array}{l}\text { Audio } \\
+ \\
\text { Video }\end{array}$ & $\begin{array}{l}\text { Fast Up / } \\
\text { Slow Down } \\
\text { (Linear } \\
\text { AMP) }\end{array}$ & $\begin{array}{c}\text { Web-based } \\
\text { VoD }\end{array}$ & $\begin{array}{c}\text { QoE } \\
\text { (Crowsourcing) }\end{array}$ & $\begin{array}{l}\text { Big } \\
\text { Bunny } \\
\text { (animation/cart } \\
\text { oons, no natural } \\
\text { actions and } \\
\text { speech) }\end{array}$ \\
\hline $\begin{array}{l}\text { This } \\
\text { work }\end{array}$ & $\begin{array}{l}\text { Any (but described } \\
\text { and evaluated } \\
\text { within the context } \\
\text { of IDMS) }\end{array}$ & $\begin{array}{l}\text { Any (but } \\
\text { described and } \\
\text { evaluated } \\
\text { within the } \\
\text { context of } \\
\text { asynchrony } \\
\text { between } \\
\text { receivers) }\end{array}$ & $\begin{array}{l}\text { Audio } \\
+ \\
\text { Video }\end{array}$ & $\begin{array}{l}\text { Fast Up / } \\
\text { Slow Down } \\
\text { (Cubic } \\
\text { AMP) }\end{array}$ & $\begin{array}{l}\text { Web-based } \\
\text { VoD }\end{array}$ & $\begin{array}{c}\text { QoS (real } \\
\text { scenario) + QoE } \\
\text { tests (controlled } \\
\text { lab studies) }\end{array}$ & $\begin{array}{l}\text { Sports-related } \\
\text { documentary, } \\
\text { with natural } \\
\text { speech and } \\
\text { action scenes }\end{array}$ \\
\hline
\end{tabular}

${ }^{a}$ Identification of content sections with specific audio/visual features (e.g., slow motion scenes or low audio intensity). It is also referred to as context-aware in [10], since this approach minimizes the chances of noticeability of the playout adjustments. 
In [3], the impact of using aggressive playout adjustment techniques (i.e., skips and pauses) compared to using Linear AMP techniques on the quality of lip-sync in (live) media streaming services is objectively and subjectively evaluated. Lip-sync is the most common example of intermedia sync, and involves the sync between the spoken voice and the associated movements of the speakers' lips (i.e., video). To ensure a high quality lip-sync, both intra-media and inter-media sync need to be provided. Likewise, when performing lip-sync, the audio stream is generally taken as the sync reference to which the video stream must synchronize, as the human perception is more sensitive to audio adjustments than to video adjustments. However, for simplicity, in that work, it is assumed that intra-media is already guaranteed for the (master) audio stream and that only intermedia sync control needs to be performed for the (slave) video stream. The results of the objective evaluations showed that the use of Linear AMP techniques results in a significantly better performance and QoS, in terms of average MU rate (as no MUs are discarded when using AMP), total pause time (as no MUs are paused when using AMP) and of inter-media sync accuracy, compared to the use of aggressive adjustment techniques. It is also shown that the differences become more relevant as the network delays and jitter increase. The results of the subjective evaluations demonstrated that the use of Linear AMP techniques results in a significantly better QoE than the use of aggressive adjustment techniques, and, again, that the differences become more relevant as the network delays and jitter increase.

In [6], it is shown that the coordinated operation between a proposed Linear AMP strategy and a proposed packet re-transmission strategy allows reducing the streaming delays (by buffering less amount of data) and the probability of buffer underflow situations (by slowing down the playout rate when the buffer occupancy is below a certain threshold) in video streaming services over errorprone channels. The evaluation methodology is based on simulation experiments, by modeling the overall system, adopting two-state Markov processes to model the packet loss probability for the transmission channel and the packet re-transmission strategy. In the evaluations, it is assumed that all MUs (concretely, the video frames) have the same size and that the transmitted packets contain just one frame, which is not very realistic in real contemporary multimedia systems.

In [7], it is presented a model-based and content-aware Linear AMP solution for mobile devices in wireless video streaming services. It makes use of statistical assumptions of both the arrival and departure processes for a better decision on the dynamic threshold and on the magnitude of the playout rate adjustments. It is content-aware because the (syntax-level) information about the video frame sizes is taken into account when calculating the required playout adjustments.

In [8], it is proposed a cross-layer solution, based on the coordination between a server-based packet scheduling strategy - at the Medium Access Control (MAC) layer - and a client-based Linear AMP strategy - at the application layer -, to maximize the perceived video quality in streaming services over wireless links, overcoming potential variations of the channel conditions. At the server side, the packet scheduling strategy may decide to transmit the most important MUs 
and just skip some others during periods in which the bandwidth is scarce, avoiding the transmission of packets that may be lost because of poor channel conditions or may likely be discarded at the client side because of late arrival. At the client side, the AMP strategy may decide to (constantly) slow down the playout rate in order to still accommodate the playout of late packets during periods in which the channel conditions are not adequate, preventing from buffer underflow situations and trying to ensure the media playout continuity. The proposed cross-layer solution is also content-aware. At the server side, packets are selectively discarded to meet the rate constraints, with the lowest impact as possible on the media distortion. At the client side, the motion characteristics (e.g., intensity) of the video scenes are taken into account when adjusting the playout rate. The idea is to preferentially adjust the playout rate in scenes with slow or no motion, as it may be less annoying to the human perception than doing so in scenes with high motion. The overall system is modeled, including the video quality distortion at the server side, as well as variable bandwidth and delays at the distribution side (wireless environment). Packet loss is not considered, as it is assumed that the physical and MAC layers make use of encoding, retransmissions or Forward Error Correction (FEC) techniques to mitigate such effects. The system is evaluated by using real frameworks for the server and client sides, but simulating the conditions of the wireless channel. The system performance is objectively evaluated, taking into account the following metrics: playout distortion, playout duration and video quality (using the Peak Signal-toNoise Ratio - PSNR - metric). Moreover, subjective tests were conducted, which mainly confirmed that: 1) the perceived video quality degrades when (constantly) slowing down the playout rate, but it is much more preferable than the occurrence of media playout disruptions; and 2) slowing down the playout rate in scenes with low motion intensity is more tolerable to the human perception than doing so in high motion scenes. However, few details about these subjective assessments are provided. Unlike in [6], in which the relevant parameters for AMP, such as the buffer occupancy thresholds and the playout rate variation factors are pre-specified, that works adopts a controlled Markov chain to dynamically adjust the values of and limits for these parameters according to the channel conditions.

The work in [9] proposed another Linear AMP solution that takes into account the buffer fullness variation as the criteria for determining the need for adjusting the playout process (i.e., for triggering the AMP process), rather than the buffer fullness levels, as in other works (e.g., [6] and [7]). Simulation results show that the proposed AMP solution is able to avoid buffer outage, provides better video quality (measured in terms of the variation of the playout duration) and it is less sensitive to variable network conditions than other existing solutions. That work focuses on the application of AMP for video streaming services, without including audio. Similarly than in [6], the overall system is modeled, including two-state Markov processes to model the packet error probability and a packet re-transmission strategy. 
In [1], a Linear AMP solution is proposed to enhance the performance of a standard IDMS solution, by achieving a higher sync accuracy and minimizing the occurrence of long-term (and noticeable) playout discontinuities (i.e., skips and pauses). In [2], the previous work is extended, by demonstrating that the use of Linear AMP is beneficial when using different architectural schemes to exchange the control information and different strategies to choose the reference to synchronize with in IDMS-enabled systems.

In [5], an arrival process-controlled AMP solution with multiple thresholds to improve the playout quality in video streaming services is proposed. The first threshold is used in the pre-roll period to calculate the initial playout instant, based on the jitter for the incoming video frames. Additionally, two thresholds for the upper and lower buffer fullness levels are used during the playout period. If the buffer fullness level is below or over these upper and lower thresholds, the AMP process is triggered to either fast up or slow down the playout rate, respectively, in order to restore the buffer occupancy into safe ranges. The novelty of the proposed solution resides in that the playout process is adjusted in a quadratic manner (i.e., Quadratic AMP), and not in a linear (i.e., constant) manner (i.e., Linear AMP), as in all the AMP solutions proposed in the other mentioned works. When the buffer fullness level is within these two thresholds, the playout rate will depend on the instantaneous frame arrival rate, which is estimated by using a proposed arrival process tracking algorithm. Theoretical analysis and simulation results show the benefits of the proposed solutions, in terms of reducing the initial playout delay, and avoiding buffer underflow and overflow situation. In addition, it is shown that the proposed Quadratic AMP solution outperforms traditional Linear AMP solutions (such as the ones proposed in [6] and in [9]) in terms of the smoothness of the playout curve, by using the variance of distortion of playout metric (also used in this work and defined in Section 4.1). However, no subjective assessments were conducted to corroborate the benefits on the perceived QoE.

In [10], the impact on the perceived QoE of the application of two different Linear AMP solutions was evaluated and compared by adopting a crowdsourcing-based methodology. The first AMP solution randomly chooses content sections for increasing and decreasing the playout rate. The second AMP solution takes into account audio-visual features for identifying appropriate content sections for which the playout rate may be increased or decreased, being therefore contentaware. Regarding the audio-visual features, the average length of motion vectors between consecutive frames (i.e., the motion intensity) is selected for video, and the Root Mean Square (RMS) of the envelope of each frame (i.e., the audio intensity) is selected for audio. The aim is to trigger the playout adjustments in these content sections in which the probability of noticeability may be lower, thus minimizing the impact on the QoE, with the assumption that it is preferable to slow down the playout rate in high motion scenes with low audio volume, and to fast up the playout rate in slow motion scenes with low audio volume. The evaluations were performed for different ranges of constant playout rate rations (i.e., Linear AMP) during the same period interval 
using the two AMP solutions. They revealed that there is no significant difference between both solutions for slight playout rate variations, but that the content-aware AMP solution performs significantly better in extreme situations in which the playout rate is very low or very high. Interestingly, it was found that increasing the playout rate does not significantly degrade the perceived QoE if the content sections in which to perform the playout adjustments (e.g., with no speech, low audio volume or slow motion video scenes) are properly selected. Therefore, the decision on when to perform the playout adjustment plays a key role. In that study, it was also noticed that decreasing the playout rate results into higher QoE degradations than increasing the playout rate by the reciprocal factor of the decrease. The application area of that work was IDMS, but the video sequences were evaluated by individual users, and not in the context of a shared experience.

In [11], the impact on the QoE of increasing and decreasing the playback rate for randomly selected content sections of different duration is subjectively assessed, by using crowdsourcing. In that study, the audio-visual features from [10] are used as metrics to quantify the audio and video distortions caused by the (constant) playout rate variations. Then, a non-linear model is analytically derived by correlating the values of these metrics and the results of the subjective assessments, with the goal of estimating the QoE by analyzing the audiovisual contents (i.e., the magnitudes of audio and video distortion measured by using the above metrics). The subjective assessments revealed interesting findings. First, it is shown that small distortions in these metrics, especially for audio, already have an impact on the QoE. Second, adjusting the playout rate for the combination of audio and video has a higher impact on the QoE than adjusting the playout rate for only video (as done in other works e.g. [7, 9]), as playout adjustments for audio are more critical to the human perception than playout adjustments for video. Third, and corroborating the results in [10], increasing the playout rate for specific content sections has a lower impact on the QoE than decreasing the playout rate for the same sections by the reciprocal of the increase of the playback rate.

\section{AMP Strategies}

This Section initially introduces the AMP process, involving a deviated playout curve and a reference one, together with the relevant aspects of, and parameters in, such a process. Next, it provides a mathematical formulation and analysis of the interpolation curves between the deviated and reference playout curves when using the existing Linear and Quadratic AMP strategies. The duration of the adjustment period when bounding the maximum value of the playout rate variation to $\varphi_{m a}$ in each one of them is also derived. Then, the same expressions are formulated for the proposed strategy in this work, Cubic AMP. Finally, the Linear, Quadratic and Cubic AMP strategies are compared in terms of the computational complexity, smoothness of the playout curve and of the duration of the adjustment period. It is also discussed why higher-order polynomial interpolation methods are less appropriate than Cubic AMP. 


\subsection{Modeling the AMP Process}

Let us consider an example consisting of a reference playout curve (Eq. 1), with an ideal playout rate of $\theta \mathrm{MU} / \mathrm{s}$, and a deviated curve ${ }^{2}$ (Eq. 2), with a playout rate of $\mu=\theta \cdot(1+\varphi) \mathrm{MU} / \mathrm{s}$, being $\mu<\theta$. These playout curves are represented in Figures 1 and 2.

$$
\begin{aligned}
& p_{\text {ref }}(t)=\theta \cdot t \\
& p_{\text {dev }}(t)=\mu \cdot t
\end{aligned}
$$

If no correction is applied, the asynchrony between both playout processes would progressively increase, with a consequent QoE degradation. For example, if both playout processes start simultaneously at $t_{i n i}=0 \mathrm{~s}$, then an allowed asynchrony threshold of $\Delta \tau$ MUs between both playout processes will be reached at $t_{\tau}=\Delta \tau /(\mu-\theta)_{s}$. At that moment, the deviated curve must be adjusted in order to eliminate such asynchrony. If using AMP, it can be done by either speeding up (if the playout process is lagged compared to the reference), or slowing down the playout rate (if the playout process is advanced compared to the reference), as shown in Figure 1.b. This way, the deviated curve will match the reference curve $(\theta \cdot t)$ at $t_{\text {sync }}=\left(t_{\tau}+\Delta T\right) \mathrm{s}$, being $\Delta T$ the duration of the adjustment period.

In such process, three key factors must be taken into account. The first one is the upper bound within which the playout rate can be varied without degrading the QoE. A value of $\varphi_{\max }=25 \%$ is commonly adopted in literature (e.g., $[1,2,6,9]$ ), even though recent studies have put into doubt this limit and open the door to future research on this matter [10,11]. Anyway, it seems clear that an upper value for $\varphi_{\max }$ needs to be bounded for a good QoE. The second one is the duration of the adjustment process $(\Delta T)$, as it also impacts the perceived QoE [4]. The third one is the specific interpolation method between the involved (deviated and reference) curves, given specific values of $\Delta \tau, \mu, \theta$, and of either $\varphi_{\max }$ or $\Delta T$.

The next three sub-sections provide the expressions of the interpolation curves and of the duration of the adjustment periods for the existing Linear and Quadratic AMP strategies and, most importantly, for the proposed Cubic AMP strategy. For each one of the AMP strategies, the initial and final points of the interpolation curves are given by $Q_{\tau}=\left(t_{\tau}, \mu \cdot t_{\tau}\right)$ and $Q_{\text {sync }}=\left(t_{\text {sync }}, \theta \cdot t_{\text {sync }}\right)$, respectively (see Figure 1.b).

\subsection{Linear $A M P$}

Using Linear AMP, a linear interpolation curve (Eq.3) between the deviated curve (Eq. 1) at $t=t_{\tau}$ and reference curve (Eq. 2) at $t=t_{\text {sync }}$ is employed:

$$
p_{\text {int }}^{\text {Linear }}(t)=a \cdot t+b
$$

\footnotetext{
${ }^{2}$ In this paper, without loss of generality, only playout rate skews $(\varphi)$ are considered, as this factor has a bigger impact on the deviation of the playout points. Likewise, the provided example is for a negative skew, with results in $\mu<\theta$, therefore having a lagged playout process compared to the reference. The same behavior applies for positive skews.
} 
where $a$ and $b$ are two real parameters. The linear polynomial interpolation curve must start at $Q_{\tau}$ point (matching the deviated curve) and end at $Q_{s y n c}$ point (matching the reference curve). Therefore, it has to meet the next two conditions:

$$
\begin{gathered}
p_{\text {int }}^{\text {Linear }}\left(t_{\tau}\right)=a \cdot t_{\tau}+b=p_{\text {dev }}\left(t_{\tau}\right)=\mu \cdot t_{\tau} \\
p_{\text {int }}^{\text {Linear }}\left(t_{\text {sync }}\right)=a \cdot t_{\text {sync }}+b=p_{\text {ref }}\left(t_{\text {sync }}\right)=\theta \cdot t_{\text {sync }}
\end{gathered}
$$

which is a linear system with a unique solution for the parameters, obtaining:

$$
p_{\text {int }}^{\text {Linear }}(t)=\mu \cdot t_{\tau}+\frac{t-t_{\tau}}{t_{\text {sync }}-t_{\tau}} \cdot\left(\theta \cdot t_{\text {sync }}-\mu \cdot t_{\tau}\right), t \in\left[t_{\tau}, t_{\text {sync }}\right]
$$

Apart from accurately matching the reference playout point at $t_{s y n c}$ instant, the playout rate (i.e., the derivative) of the linear interpolation playout curve must be bounded throughout the AMP process (i.e., $\left.\mu_{\max } \leq \mu \cdot\left(1+\left|\varphi_{\max }\right|\right)\right)$ to avoid too aggressive playout adjustments, which may be noticeable or even annoying to the users' perception. By imposing this condition, the value of the required duration of the adjustment period $(\Delta T)$ and, therefore, the value of $t_{s y n c}$, that guarantees that the playout rate will be lower than the upper threshold throughout the AMP process will be determined by the slope (i.e., derivative) of the interpolation curve, setting it to the maximum allowable value of the playout rate:

$$
p_{\text {int }}^{\prime \text { Linear }}(t)=\frac{\theta \cdot t_{\text {sync }}-\mu \cdot t_{\tau}}{t_{\text {sync }}-t_{\tau}}=\frac{\theta \cdot \Delta T+(\theta-\mu) \cdot t_{\tau}}{\Delta T}=\mu \cdot\left(1+\varphi_{\max }\right)
$$

The above expression is a linear equation in $\Delta T$ where the term $(\theta-\mu) \cdot t_{\tau}$ is the allowed asynchrony threshold $\Delta \tau$, so the expression for the adjustment period can be written as:

$$
\Delta T_{\text {Linear }}=\frac{\Delta \tau}{\mu \cdot\left(1+\varphi_{\max }\right)-\theta}
$$

To achieve that the playout rate variation will be lower than $\varphi_{\max }$ throughout the adjustment process when using Linear AMP, the value of $\Delta T_{\text {Linear }}$ must be applied to Eq. 5 ( $t_{\tau}$ is known, and $\left.t_{\text {sync }}=t_{\tau}+\Delta T_{\text {Linear }}\right)$.

\subsection{Quadratic AMP.}

Using Quadratic AMP, a quadratic (i.e., parabolic) interpolation curve (Eq.8) between the deviated (Eq. 1) curve and reference curve (Eq. 2) is employed. It can be written as:

$$
p_{\mathrm{int}}^{\text {Quad }}(t)=a \cdot t^{2}+b \cdot t+c
$$

where $a, b$ and $c$ are three real parameters. However, for computational simplicity, it is much more convenient to write the above expression by making use of a new temporal variable $\zeta=\left(t-t_{\tau}\right) /\left(t_{\text {sync }}{ }^{-}\right.$ $\left.t_{\tau}\right)$ :

$$
p_{\text {int }}^{\text {Quad }}(\zeta)=a \cdot \zeta^{2}+b \cdot \zeta+c, \text { where } \zeta=\frac{t-t_{\tau}}{t_{\text {sync }}-t_{\tau}} \in[0,1]
$$


where $a, b$ and $c$ are (the new) three real parameters. With this new variable, when $t=t_{\tau} \rightarrow \zeta=0$, when $t=t_{\text {sync }} \rightarrow \zeta=1$, and the time derivative can be written as:

$$
\frac{d p}{d t}(\zeta)=\frac{d p}{d \zeta} \frac{d \zeta}{d t}=\frac{1}{t_{\text {sync }}-t_{\tau}} \frac{d p}{d \zeta}
$$

As in the linear case, the quadratic polynomial interpolation curve must start at $Q_{\tau}$ point (matching the deviated curve) and end at $Q_{\text {sync }}$ point (matching the reference curve).

Apart from these two conditions, a third one has to be fixed, which can be the derivative of the playout curve (i.e., the rate) at either the beginning or the end of the adjustment process (see Figures 2.b1 and 2.b2, respectively) [5], but not at both. As an example, by fixing it at the beginning of the adjustment process, these three conditions can be formulated as

$$
\begin{aligned}
& p_{\text {int }}^{\text {Quad }}(0)=c=\mu \cdot t_{\tau} \\
& p_{\text {int }}^{\text {Quad }}(1)=a+b+c=p_{\text {ref }}\left(t_{\text {sync }}\right)=\theta \cdot t_{\text {sync }} \\
& \frac{d p_{\text {int }}^{\text {Quad }}}{d t}(0)=\frac{1}{t_{\text {sync }}-t_{\tau}} \cdot b=p_{\text {dev }}^{\prime}\left(t_{\tau}\right)=\mu
\end{aligned}
$$

a) Linear AMP

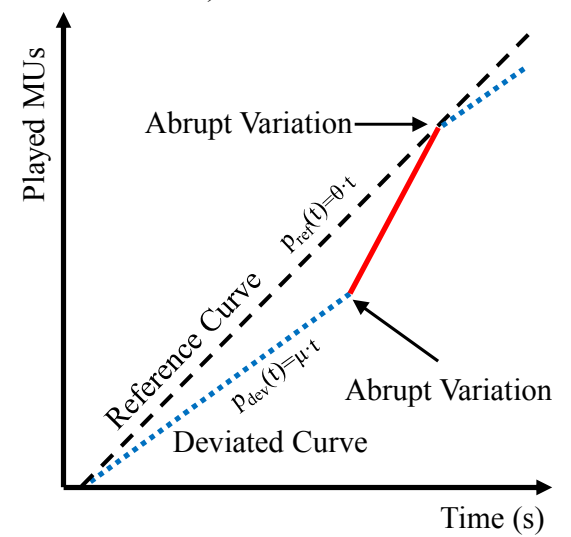

b2) Quadratic AMP

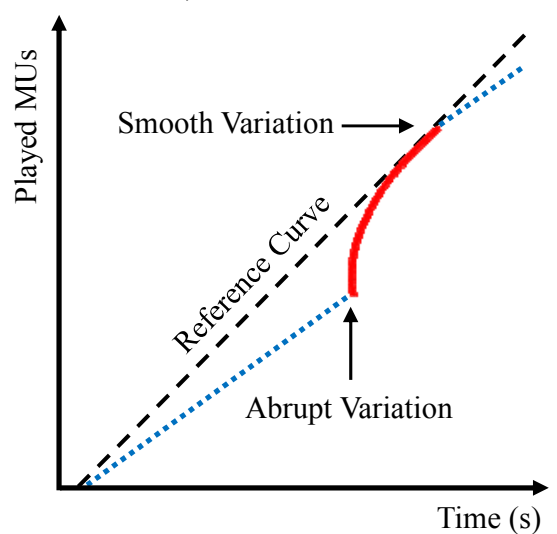

b1) Quadratic AMP

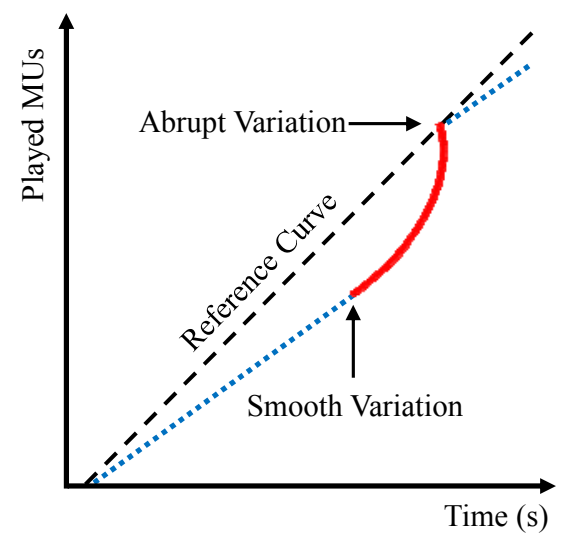

c) Cubic AMP

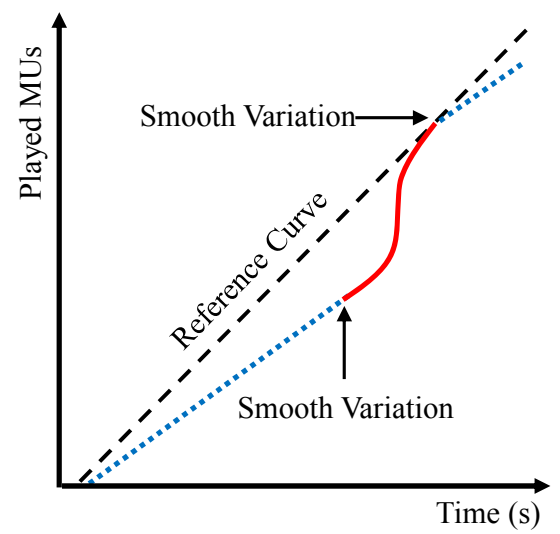

Figure 2. Interpolation methods when using the different AMP strategies for lagged playout curves. The same behavior applies for advanced playout curves. 
This is a linear system with a unique solution for the parameters, which can be solved straightforward, obtaining:

$$
p_{\text {int }}^{\text {Quad }}(\zeta)=(\theta-\mu) \cdot t_{\text {sync }} \cdot \zeta^{2}+\mu \cdot\left(t_{\text {sync }}-t_{\tau}\right) \cdot \zeta+\mu \cdot t_{\tau}, \text { where } \zeta=\frac{t-t_{\tau}}{t_{\text {sync }}-t_{\tau}} \in[0,1]
$$

As in Linear AMP, the playout rate (i.e., the derivative) of the quadratic interpolation playout curve must be bounded throughout the AMP process (i.e., $\left.\mu_{\max } \leq \mu \cdot\left(1+\left|\varphi_{\max }\right|\right)\right)$. By imposing this condition, the value of $\Delta T$ (and, therefore, the value of $t_{s y n c}$ ) will be determined by the slope of the quadratic interpolation curve, which needs to be bounded in its maximum point. Therefore, the maximum point needs to be found and the value of the slope in this point has to be fixed to $\mu \cdot\left(1+\varphi_{\max }\right)$.

For that purpose, it is firstly checked that the playout rate $d p_{\text {int }}^{\text {Quad }}(\zeta) / d t$ has not a local maximum when $\zeta \in] 0,1[$, because its derivative is non-zero:

$$
\begin{aligned}
& \frac{d}{d t}\left(\frac{d p_{\text {int }}^{\text {Quad }}}{d t}\right)(\zeta)=\frac{1}{\left(t_{\text {sync }}-t_{\tau}\right)^{2}} \cdot \frac{d^{2} p_{\text {int }}^{\text {Quad }}}{d \zeta^{2}}(\zeta) \\
& =\frac{1}{\left(t_{\text {sync }}-t_{\tau}\right)^{2}} \cdot \frac{d}{d \zeta} \cdot\left(2 \cdot(\theta-\mu) \cdot t_{\text {sync }} \cdot \zeta+\mu \cdot\left(t_{\text {sync }}-t_{\tau}\right)\right)=\frac{2 \cdot(\theta-\mu) \cdot t_{\text {sync }}}{\left(t_{\text {sync }}-t_{\tau}\right)^{2}} \neq 0
\end{aligned}
$$

Then, the maximum slope occurs in one of the two ends of the playout adjustment interval. As

$$
\frac{d p_{\mathrm{int}}^{\text {Quad }}}{d t}(0)=\mu<\frac{d p_{\mathrm{int}}^{\text {Quad }}}{d t}(1)=\frac{2 \cdot(\theta-\mu) \cdot t_{\text {sync }}}{\left(t_{\text {sync }}-t_{\tau}\right)}+\mu
$$

because $2 \cdot(\theta-\mu) \cdot t_{\text {sync }} /\left(t_{\text {sync }}-t_{\tau}\right)>0$, the playout rate has it maximum at $\zeta_{\max }=1$. By setting the value of the slope to $\mu \cdot\left(1+\varphi_{\max }\right)$ in the maximum point $\zeta_{\max }$ :

$$
\frac{d p_{\text {int }}^{\text {Quad }}}{d t}(1)=\frac{1}{t_{\text {sync }}-t_{\tau}} \frac{d p_{\text {int }}^{\text {Quad }}}{d \zeta}(1)=\frac{2 \cdot(\theta-\mu) \cdot t_{\text {sync }}+\mu \cdot\left(t_{\text {sync }}-t_{\tau}\right)}{t_{\text {sync }}-t_{\tau}}=\mu \cdot\left(1+\varphi_{\max }\right)
$$

Using $t_{\text {sync }}=\left(t_{\tau}+\Delta T\right)$, the previous equation can be rewritten as

$$
\frac{2 \cdot(\theta-\mu) \cdot t_{\tau}+(2 \cdot \theta-\mu) \cdot \Delta T}{\Delta T}=\mu \cdot\left(1+\varphi_{\max }\right)
$$

As $(\theta-\mu) \cdot t_{\tau}$ is the allowed asynchrony threshold, $\Delta \tau$, the expression for the adjustment period can be obtained from the previous linear equation in $\Delta T$ as:

$$
\Delta T_{\text {Quad }}=\frac{2 \cdot \Delta \tau}{\varphi_{\max } \mu-2(\theta-\mu)}=\frac{\Delta \tau}{\mu \cdot\left(1+\frac{1}{2} \varphi_{\max }\right)-\theta}
$$

\subsection{Cubic AMP}

Our proposal consists of employing a cubic interpolation method (Eq. 18) between the deviated playout curve (Eq. 1) and reference playout curve (Eq. 2): 


$$
p_{\mathrm{int}}^{\text {Cubic }}(t)=a \cdot t^{3}+b \cdot t^{2}+c \cdot t+d
$$

where $a, b, c$ and $d$ are four real parameters. However, as in Quadratic AMP, for computational simplicity, it is much more convenient to write the above expression and the system by making use of a new temporal variable $\zeta=\left(t-t_{\tau}\right) /\left(t_{\text {sync }}-t_{\tau}\right)$, resulting in:

$$
p_{\text {int }}^{\text {Cubic }}(\zeta)=a \cdot \zeta^{3}+b \cdot \zeta^{2}+c \cdot \zeta+d, \text { where } \zeta=\frac{t-t_{\tau}}{t_{\text {sync }}-t_{\tau}} \in[0,1]
$$

where $a, b, c$ and $d$ are (the new) four real parameters.

As in the previous strategies, the cubic polynomial interpolation curve must start at $Q_{\tau}$ point (matching the deviated curve) and end at $Q_{s y n c}$ point (matching the reference curve). Apart from these two conditions, two additional ones have to be fixed, which are the derivatives of the playout curve (i.e., the rate) at the beginning and the end of the adjustment process. This will ensure a smooth transition between the involved curves, avoiding bends, as can be seen in Figure 2.c.

Taking into account Eq. 10 and that when $t=t_{\tau} \rightarrow \zeta=0$, and when $t=t_{\text {sync }} \rightarrow \zeta=1$, the linear system that determines the parameters can now be written as:

$$
\begin{aligned}
& p_{\text {int }}^{\text {Cubic }}(0)=d=p_{\text {dev }}\left(t_{\tau}\right)=\mu \cdot t_{\tau} \\
& p_{\text {int }}^{\text {Cubic }}(1)=a+b+c+d=p_{\text {ref }}\left(t_{\text {sync }}\right)=\theta \cdot t_{\text {sync }} \\
& \frac{d p_{\text {int }}^{\text {Cubic }}}{d t}(0)=\frac{1}{t_{\text {sync }}-t_{\tau}} \cdot c=p^{\prime}{ }_{d e v}\left(t_{\tau}\right)=\mu \\
& \frac{d p_{\text {int }}^{\text {Cubic }}}{d t}(1)=\frac{1}{t_{\text {sync }}-t_{\tau}} \cdot(3 a+2 b+c)=p_{\text {dev }}^{\prime}\left(t_{\text {sync }}\right)=\theta
\end{aligned}
$$

Thanks to the definition and use of the new temporal variable, it is not difficult to solve this system, obtaining:

$$
\begin{aligned}
p_{\mathrm{int}}^{\text {Cubic }}(\zeta) & =-(\theta-\mu) \cdot\left(t_{\text {sync }}+t_{\tau}\right) \cdot \zeta^{3}+(\theta-\mu) \cdot\left(2 \cdot t_{\text {sync }}+t_{\tau}\right) \cdot \zeta^{2} \\
& +\mu \cdot\left(t_{\text {sync }}-t_{\tau}\right) \cdot \zeta+\mu \cdot t_{\tau}, \text { where } \zeta=\frac{t-t_{\tau}}{t_{\text {sync }}-t_{\tau}} \in[0,1]
\end{aligned}
$$

As in the other AMP strategies, the playout rate (i.e., the derivative) of the interpolation playout curve must be bounded throughout the AMP process (i.e., $\mu_{\max } \leq \mu \cdot\left(1+\left|\varphi_{\max }\right|\right)$. By imposing this condition, the value of $\Delta T$ (and, therefore, the value of $t_{\text {sync }}$ ) will be determined by the slope of the cubic interpolation curve, which needs to be bounded in its maximum point.

Therefore, the maximum point needs to be found and the value of the slope in this point has to be fixed to $\mu \cdot\left(1+\varphi_{\max }\right)$. For that purpose, we equal to zero the first derivative, because we want to find any maximum $\zeta_{\max }$ in $] 0,1[$ :

$$
\frac{d}{d t}\left(\frac{d p_{\text {int }}^{\text {Cubic }}}{d t}\right)\left(\zeta_{\max }\right)=\frac{1}{\left(t_{\text {sync }}-t_{\tau}\right)^{2}} \cdot \frac{d^{2} p_{\text {int }}^{\text {Cubic }}}{d \zeta^{2}}(\zeta)=0
$$

which is the same as 


$$
\begin{aligned}
& \frac{d^{2} p_{\text {int }}^{\text {Cubic }}}{d \zeta^{2}}(\zeta)=\frac{d}{d \zeta}\left(-3 \cdot(\theta-\mu) \cdot\left(t_{\tau}+t_{\text {sync }}\right) \cdot \zeta^{2}+2 \cdot(\theta-\mu) \cdot\left(t_{\tau}+2 t_{\text {sync }}\right) \cdot \zeta\right. \\
& \left.\quad+\mu \cdot\left(t_{\text {sync }}-t_{\tau}\right)\right)=-6 \cdot(\theta-\mu) \cdot\left(t_{\tau}+t_{\text {sync }}\right) \cdot \zeta+2 \cdot(\theta-\mu) \cdot\left(t_{\tau}+2 t_{\text {sync }}\right)=0
\end{aligned}
$$

From the above expression, the value of $\zeta_{\max }$ is:

$$
\zeta_{\max }=\frac{t_{\tau}+2 t_{\text {sync }}}{3 \cdot\left(t_{\tau}+t_{\text {sync }}\right)}
$$

The playout rate at $\zeta=0$ is $\mu$ and at $\zeta=1$ is $\theta$, because of the two last conditions in Eq. 20. Then, the maximum value of the playout rate $\mu \cdot\left(1+\varphi_{\max }\right)$ is fixed in the extremum $\zeta_{\max }$ in $] 0,1[$,

$$
\begin{aligned}
& \mu \cdot\left(1+\varphi_{\max }\right)=\frac{d p_{\text {int }}^{\text {Cubic }}}{d t}\left(\zeta_{\max }\right)=\frac{1}{t_{\text {sync }}-t_{\tau}} \frac{d p_{\text {int }}^{\text {Cubic }}}{d \zeta}\left(\zeta_{\max }\right) \\
& =\frac{1}{t_{\text {sync }}-t_{\tau}} \cdot\left(-\frac{(\theta-\mu) \cdot\left(t_{\tau}+2 \cdot t_{\text {sync }}\right)^{2}}{3 \cdot\left(t_{\tau}+t_{\text {sync }}\right)}+\frac{2 \cdot(\theta-\mu) \cdot\left(t_{\tau}+2 \cdot t_{\text {sync }}\right)^{2}}{3 \cdot\left(t_{\tau}+t_{\text {sync }}\right)}+\mu \cdot\left(t_{\text {sync }}-t_{\tau}\right)\right)
\end{aligned}
$$

which gives:

$$
\frac{(\theta-\mu) \cdot\left(2 \cdot t_{s y n c}+t_{\tau}\right)^{2}}{3 \cdot\left(t_{s y n c}^{2}-t_{\tau}^{2}\right)}=\mu \cdot \varphi_{\max }
$$

Using $\Delta T=\left(t_{\text {sync }}-t_{\tau}\right)$, the above equation results in a second order polynomial in $\Delta T$, whose solution is:

$$
\Delta T_{\text {Cubic }}=3 \cdot \Delta \tau \cdot \frac{\theta-\mu \cdot\left(1+\frac{1}{2} \varphi_{\max }\right)+\frac{1}{2} \cdot \sqrt{\varphi_{\max } \cdot \mu \cdot\left(\mu \cdot\left(1+\varphi_{\max }\right)-\theta\right)}}{2 \cdot(\theta-\mu) \cdot\left(\mu \cdot\left(1+\frac{3}{4} \varphi_{\max }\right)-\theta\right)}
$$

\subsection{Comparison between the different AMP strategies: Benefits of Using Cubic AMP}

For a given value of $\varphi_{\max }$, Linear AMP is the best AMP strategy for minimizing the length of $\Delta T$, as the maximum playout rate variation is constantly applied throughout the adjustment period. However, the issue when using Linear AMP is that the playout rate is constant during the adjustment period, which originates abrupt variations or bends (obviously, depending on the value of $\varphi_{\max }$ ) at both the beginning and the end of the adjustment process (see Figure 2a). This may be noticed by users, and even be annoying to them (QoE degradation).

For the same values of $\mu, \Delta \tau$ and $\varphi_{\max }$, the duration of the adjustment period $(\Delta T)$ when using Quadratic AMP, given by Eq.17, is two times or more the one when using Linear AMP, given by Eq. 7 , i.e., $\Delta T_{\text {Quad }} \approx 2 \cdot \Delta T_{\text {Linear }}$ (see Figure 3 ). In addition, although the variation in the playout rate can be avoided at either the start or the end of the adjustment process (depending on where the derivative has been fixed), as can be seen in Figures 2.b1 and 2.b2, it will be greater than in Linear AMP in the other end. These two issues may also result in a degradation of the user's perceived QoE. 
For the same values of $\mu, \Delta \tau$ and $\varphi_{\max }$, the duration of the adjustment period $(\Delta T)$ when using Cubic AMP, given by Eq. 27, is fifty percent longer or more than the one when using Linear AMP, given by Eq. 7 , i.e., $\Delta T_{\text {Cubic }} \approx 1.5 \cdot \Delta T_{\text {Linear }}$ (see Figure 3 ). However, it is $25 \%$ shorter or more compared to when using Quadratic AMP (see Figure 3). As another graphical example, a comparison between $\Delta T_{\text {Linear }}$ and $\Delta T_{\text {Cubic }}$ for different values of $\Delta \tau$ and $\varphi_{\max }$, given a fixed value of $\mu=24.98 \mathrm{MU} / \mathrm{s}$, is provided in Figure 4.
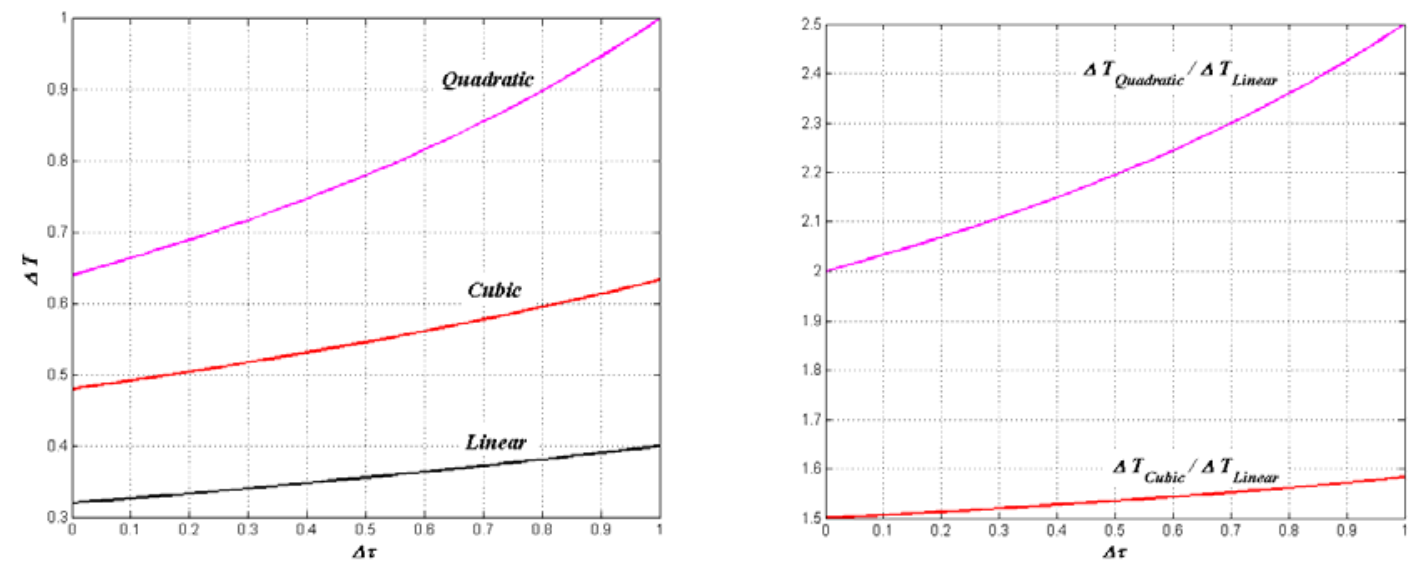

Figure 3. Comparison of $\Delta T$ versus $\Delta \tau$ for Linear, Quadratic and Cubic AMP $\left(\varphi_{\max }=0.25\right)$.

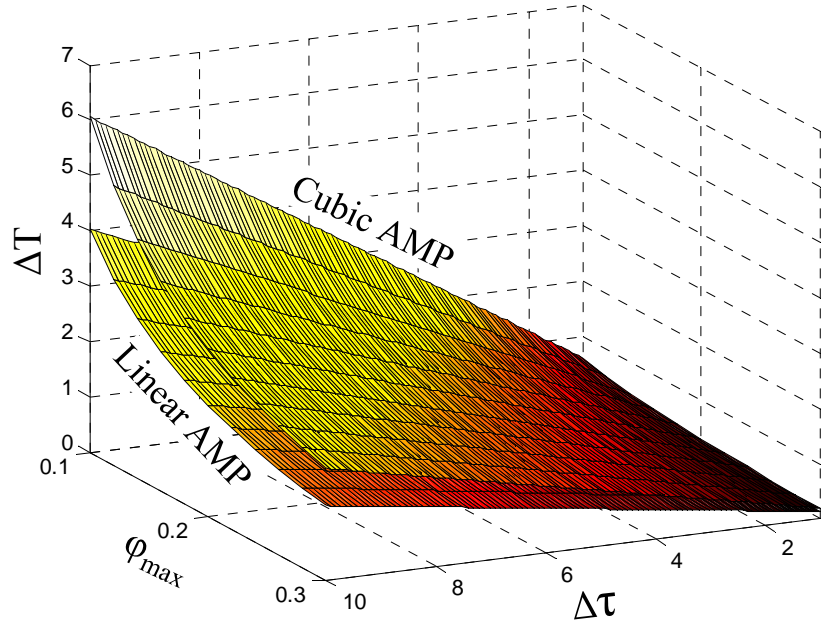

Figure 4. Comparison of $\Delta T$ when using Linear and Cubic AMP

Unlike Linear and Quadratic AMP, Cubic AMP allows avoiding bends (corners) throughout the playout adjustment process, while significantly reducing the duration of $\Delta T$ compared to Quadratic AMP, and keeping a low computational complexity, as a direct expression has been also obtained to calculate the values of both $\Delta T$ and the playout rate throughout the adjustment process.

The use of higher order polynomial and other types of non-linear and differentiable functions as the interpolation curve was also assessed. However, it involves much higher computational cost, as it requires a bigger set of equations and/or more complex mathematical methods to calculate the expressions for both the interpolation curve and $\Delta T$. In addition, it does not provide better 
performance, because Cubic AMP guarantees the continuity of the interpolation curve and of its first derivative - the playout rate -, which are the most relevant ones to avoid bends (corners).

For example, although the computational complexity for calculating the interpolation curve when using a quartic polynomial is not much higher than when using a cubic polynomial, the main difference appears when calculating the expression for $\Delta T$, because the analytical expressions of a root of a cubic polynomial (in the quartic case) are far more complex than for a quadratic polynomial (in the cubic case). Moreover, the duration of the adjustment period is thirty percent longer or more when using a quartic polynomial than when using a cubic polynomial, i.e.: $\Delta T_{\text {Quartic }}$ $\approx 1.3 \cdot \Delta T_{\text {Cubic }}$ (see supporting document). In the case of polynomial functions of higher order, it is not possible to obtain a general expression for $\Delta T$, because the roots of an arbitrary polynomial of degree higher than three cannot be written as algebraic expressions in terms of its coefficients.

In addition, it must be taken into account than when implementing the selected AMP strategy in real multimedia systems, the interpolation curve has to be discretized (e.g., per frame duration or per time interval), which also slightly increases the computational cost. Besides, the selected AMP strategy can be deployed in heterogeneous consumption devices (some of them with limited CPU resources), as well as in web browsers (with non-efficient processing and memory resources), so keeping the computational complexity very low becomes essential.

Therefore, Cubic AMP is the most suitable, and simplest, solution that meets the targeted requirements of this research work.

\section{Evaluation}

In this Section, the performance and benefits of using the proposed Cubic AMP strategy, compared to using the other existing (Linear and Quadratic) AMP strategies, are presented, both through objective evaluation (in Section 4.1) and subjective evaluation (in Section 4.2).

\subsection{Objective Evaluation (Performance and Behavior of the AMP Strategies)}

The performance and behavior of Cubic AMP, in comparison with Linear and Quadratic AMP, has been tested using Matlab ${ }^{\circledR}$. Without loss of generality, the evaluation has been focused on an IDMS scenario, which aims to guarantee synchronization between the playout processes of independent receivers. A compilation of solutions to provide IDMS, applications requiring this kind of synchronization (e.g., Social TV, multi-party conferencing...) and tolerable asynchrony thresholds in each of them can be found in [12].

Concretely, two playout processes (of two independent receivers) have been considered, assuming that the buffering strategy in use (out of the scope of this paper) in both of them was able to smooth out the effect of jitter and/or packet loss, thus preserving the original timing of the incoming media contents being played out. However, reasonable playout rate deviations were considered to (somehow) recreate the effect of congestion and/or clock imperfections, thus forcing asynchrony situations between the involved playout processes. In particular, the two playout curves 
were modeled as: $i$ ) a reference curve with an ideal playout rate of $\theta=25 \mathrm{MU} / \mathrm{s}$; and ii) a lagged curve $^{3}$ with a skew of $\varphi=-0.0008$, thus with a (deviated) playout rate of $\mu=\theta \cdot(1+\varphi)=24.98 \mathrm{MU} / \mathrm{s}$. In this case, if no correction is applied, the asynchrony between these two playout processes would reach a value of $100 \mathrm{~ms}$ in a time interval slightly superior to 2 minutes (and of $1 \mathrm{~s}$ in 20 minutes). Therefore, an allowed asynchrony threshold of $\Delta \tau=2$ MUs (i.e., $2 \cdot(1 / \theta)=80 \mathrm{~ms})$ was set to prevent from frustrating situations (bad QoE) [12]. Every time the asynchrony between both playout processes exceeds that threshold, the lagged playout process (including audio and video) must be adjusted to match the reference playout process, thus achieving synchronization between receivers (i.e., IDMS).

Figure 5 shows the interpolation processes when using each one of the AMP strategies (Linear, Quadratic and Cubic), during the same adjustment period ( $\Delta T_{\text {Linear }}$, which is the required period of time to reach the reference curve when performing a constant - linear - playout rate variation of $\varphi_{\max }=25 \%$ ) for all of them. In particular, the interpolation curves were calculated for the time interval $\left[t_{\tau}, t_{s y n c}\right]$, being $t_{\tau}=2 /(25-24.98)=100 \mathrm{~s}$, and $t_{\text {sync }}=t_{\tau}+\Delta T_{\text {Linear }}$. It can be seen that the three interpolation curves, given by Equations 5, 12 and 21, matched the reference at $t_{\text {sync }}$ s (i.e., $Q_{\text {sync }}=$ $\left.\left(t_{\text {sync }}, \theta \cdot t_{\text {sync }}\right)\right)$. Moreover, the path followed by the cubic interpolation curve was very close to the one followed by the linear interpolation curve. To check the effect of abrupt changes (i.e., bends) in the playout curves, zoom views at both the start and the end of the adjustment processes are also shown in Figure 5 (right part). It can be seen that bends at both the start and the end of the adjustment process occurred when using Linear (L) AMP. Using Quadratic (Q) AMP, the bend can be avoided at either the start or the end of the adjustment process (depending on where the derivative has been fixed). However, in the other end, it will be greater than in Linear AMP. Using Cubic (C) AMP, bends in the playout curve are avoided throughout the adjustment process.

Figure 6 shows the evolution of the derivatives of the three interpolation curves (i.e., the playout rates) during the playout adjustment process, with the goal of comparing their performance in terms of the smoothness of the playout curve, the maximum value of the playout rate (and thus, the value of the playout rate variation) and the duration of the adjustment period $(\Delta T)$. On the one hand, this figure shows the evolution of the playout rate for the three AMP strategies when considering $\Delta T_{\text {Linear }}$ (with $\varphi_{\max }=25 \%$ ) for all of them (solid lines), as in Figure 5. In this case, it can be seen that a constant playout rate variation of $\varphi_{\max }=25 \%$ was performed when using Linear (L) AMP. Therefore, abrupt changes (i.e., bends) in the playout rate occurred at both the start and the end of the adjustment process. Using Quadratic (Q) AMP, a maximum playout rate variation of $49.8 \%$ had to be performed (almost double than in Linear AMP, which was 25\%) for reaching the reference point during this time interval. Moreover, an abrupt change in the playout rate occurred at the end of the adjustment process (as discussed before, it occurs in one of the two ends), which was

\footnotetext{
${ }^{3}$ We have not considered advanced playout points in this Section. However, the behavior of the analyzed AMP strategies is identical when adjusting both advanced and lagged playout points.
} 
significantly greater than the ones when using in Linear AMP. Using Cubic (C) AMP, the playout rate was smoothly adjusted throughout the duration of the adjustment process, thus avoiding bends. In this case, the maximum playout rate variation was $37.3 \%$, which is also higher than when using Linear AMP (25\%), but significantly lower than when using Quadratic AMP (49.8\%).

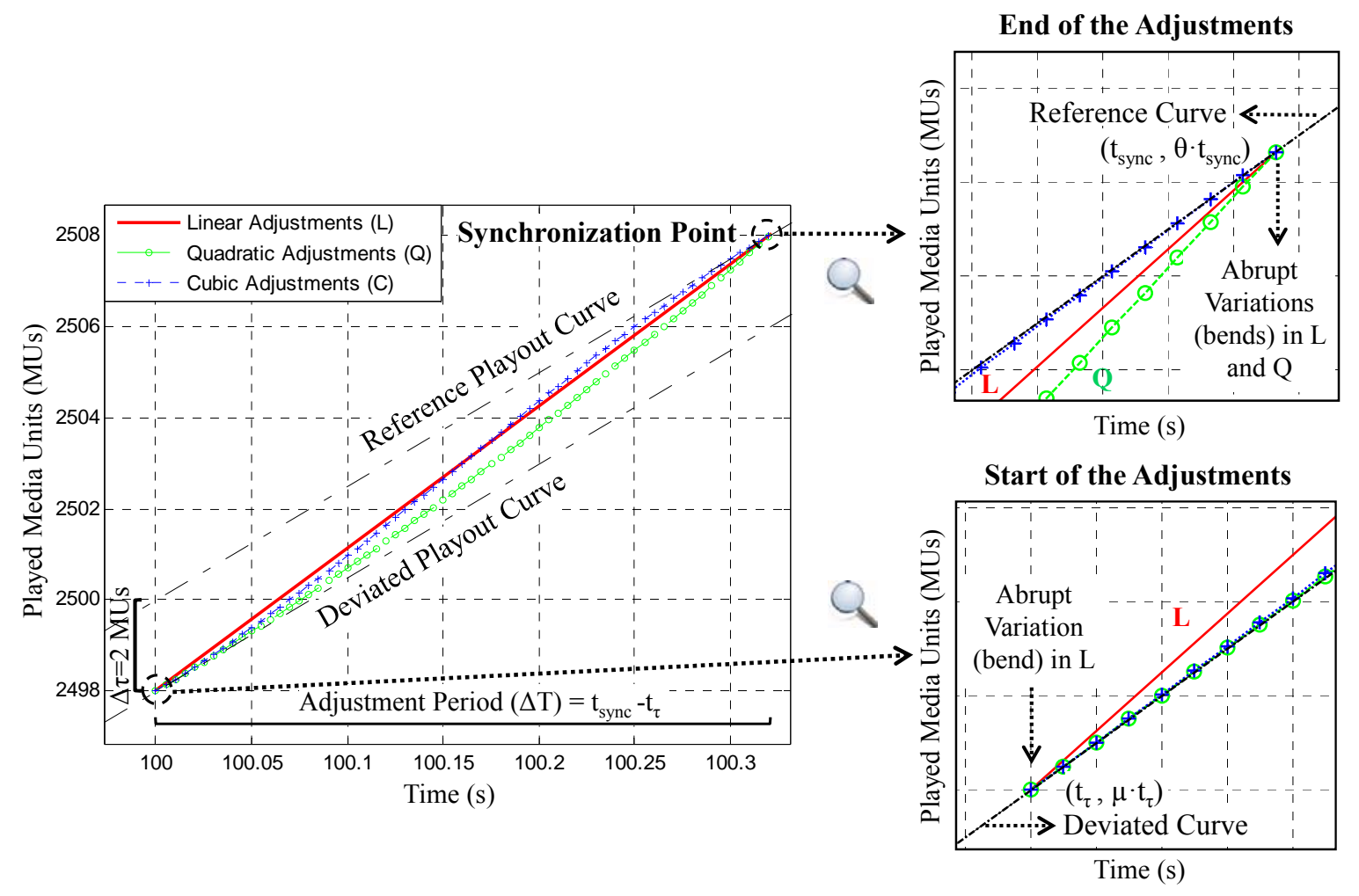

Figure 5. Interpolation curves when using each AMP strategy.

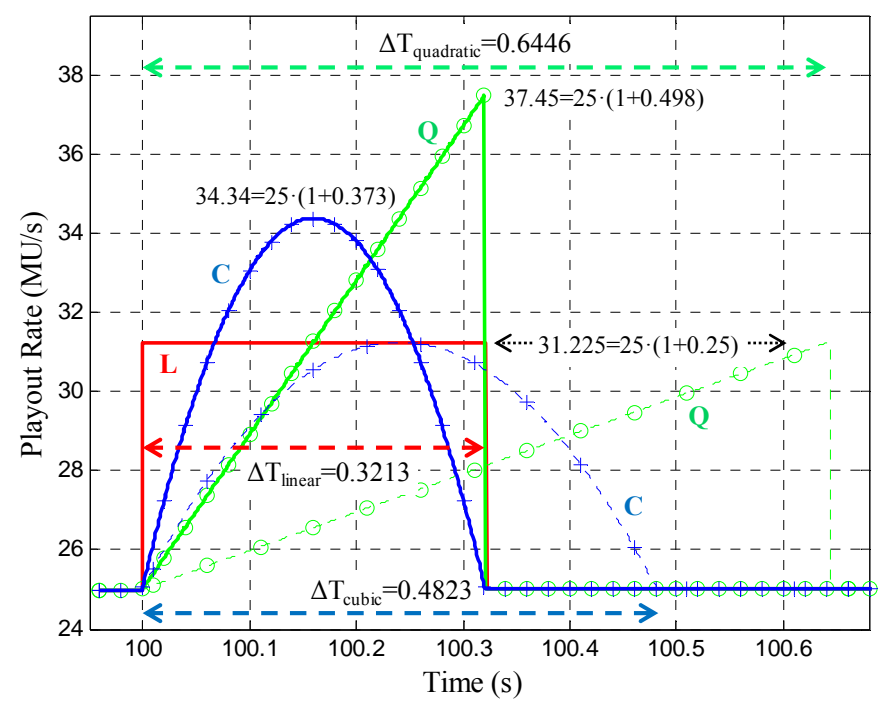

Figure 6. Playout Rate Evolution when using each AMP strategy

On the other hand, Figure 6 also represents the evolution of the playout rate curves for Quadratic and Cubic AMP when the maximum playout rate variation was bounded to $\varphi_{\max }=25 \%$ in both of them (dotted lines). It graphically confirms that Quadratic AMP requires a longer 
adjustment period to reach the reference playout curve (i.e., $\Delta T_{\text {Quadc }}>\Delta T_{\text {Cubic }}$ ), as analytically compared in Section 3. It means that Cubic AMP can recover faster from undesired situations (such as asynchrony or potential buffer underflow/overflow situations) than Quadratic AMP when fixing the same upper value of the playout rate variation in both strategies.

Although the interpolation curves are applied to the playout curve, and not to the playout rate (its first derivative), the graphs in Figure 6 show that the playout rate was also adjusted in a smoother manner when using Cubic AMP than when using Quadratic and Linear AMP, especially when bounding the playout rate variation to $\varphi_{\max }=25 \%$ (dotted blue graph). Note also that the span/zoom in Figure 6 was properly set to allow showing the evolution of the playout rates in all AMP strategies, for all conditions, in the same figure. It can be a cause why the variation of the playout rate in Cubic AMP (especially when using $\Delta T_{\text {Linear }}$ ) does not seem as smooth as it really is. In order to support this, an additional figure (Figure 7) is included, showing the evolution of the playout rate variation (i.e., the acceleration, which is the derivate of the playout rate, and therefore the second derivative of the playout curve) for the three AMP strategies. It can be seen that the acceleration when using Cubic AMP (blue curve) was also smoother than when using Linear and Quadratic AMP (for the former one, abrupt variations in the acceleration occurred at both the start and the end of the adjustment process - see red graph -, while for latter one an abrupt variation in the acceleration occurred at the end of the adjustment process - see green graph -, which was greater than for Linear AMP), avoiding abrupt changes/accelerations.

The benefits on the smoothness of the playout curve are also evaluated by considering the Variance of Distortion of Playout (VDoP) metric, given by Eq.30, as in [5]. This metric takes into account the deviation of the actual playout duration of each $n$-th frame, $S_{n}$, from the nominal one, $T=1 / \theta$, during the adjustment process:

$$
\begin{gathered}
\operatorname{DoP}(n)=\left|S_{n}-T\right| \\
\overline{D o P}=\sum_{n=1}^{N} \frac{\operatorname{DoP}(n)}{N} \\
V D o P=\frac{\sum_{n=1}^{N} \frac{D o P^{2}(n)}{N}}{N}-\overline{D o P}^{2}
\end{gathered}
$$

For the analyzed scenario, the VDoP during the adjustment when using Cubic AMP is $17.6 \%$ lower than when using Quadratic AMP, which numerically confirms that the playout curve was smoother using the proposed Cubic AMP strategy.

Therefore, Figures 5, 6 and 7 graphically show the benefits of using Cubic AMP, as it outperforms both Quadratic and Linear AMP in terms of the smoothness of the playout curve, avoiding bends throughout the adjustment process, without involving an increase of the computational complexity, and it also significantly outperforms Quadratic AMP in terms of the duration of the adjustment process (gain of $25 \%$ or even higher). 


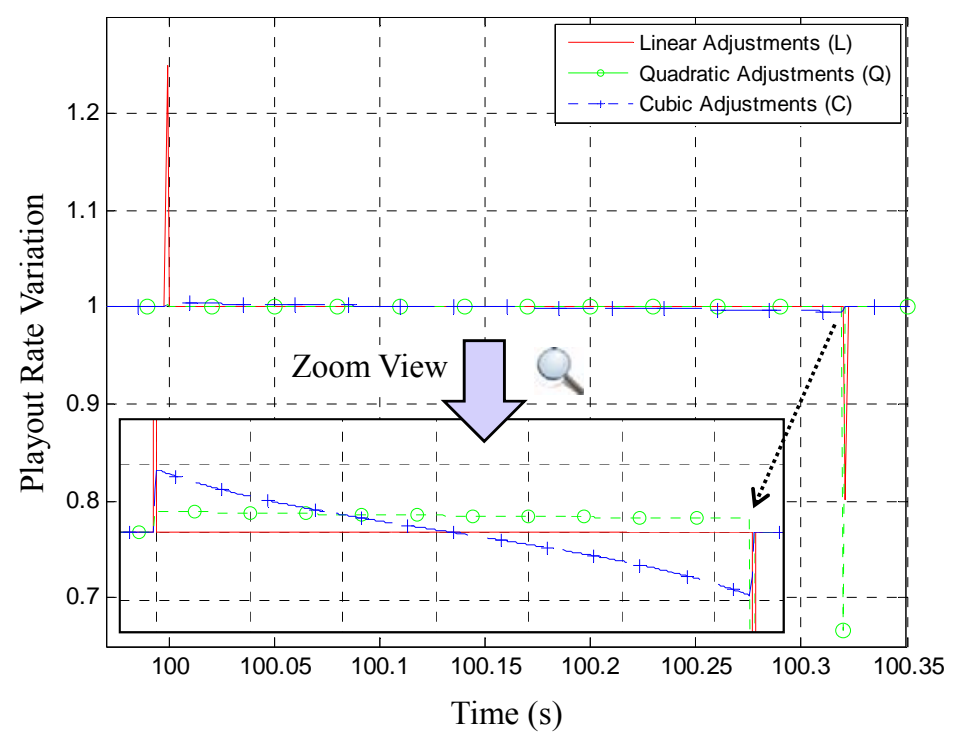

Figure 7. Evolution of the Playout Rate Variation (i.e., acceleration, second derivative of the playout curve) when using each AMP strategy

\subsection{Subjective Evaluation (QoE tests)}

The three AMP strategies have been implemented in a web-based video watching scenario (as e.g. in $[4,10,11]$, see Table 1), by using HTML5 and Javascript. It is a very relevant use case, given the ever increasing usage of online platforms (e.g., Youtube, Netflix...) for consuming media content delivered via web-based technologies. In particular, two sequences of $30 \mathrm{~s}$ of a sports related video clip were considered (with a frame rate of $\theta=25 \mathrm{MU} / \mathrm{s}$ ). The Video Sequence 1 consists of an excerpt of a first plane interview to a famous sportsman, including natural speech and human gestures. The Video Sequence 2 consists of a more action-related sequence, with faster motion, and also including natural speech, gestures and movements by the same characters.

While watching the sequences, a specific playout adjustment condition was performed at a given instant $\left(t_{\tau}\right)$, to correct a "virtual" asynchrony between the playout processes of two different receivers (including audio and video) of either $\Delta \tau=+1 \mathrm{~s}$ (receiver with an advanced playout process) or $\Delta \tau=-1 \mathrm{~s}$ (receiver with a lagged playout process), which is a commonly used threshold for Social TV scenarios [12]. Concretely, for each video sequence, 13 test conditions were considered: 5 video sequences with the playout adjustment conditions represented in Figure 4 (for both $\Delta \tau=+1 \mathrm{~s}$ or $\Delta \tau=-1 \mathrm{~s}$ ), one with a playout skip, one with a playout pause, and the (hidden) reference video sequence (i.e., the one without playout adjustments), as recommended by International Telecommunication Union (ITU) in [13].

These test conditions were randomly presented to 24 users (with gender balance, and aged between 18 and 45), who evaluated the perceived QoE for each of them, by using the Mean Opinion Score (MOS) metric, with the classical 5-point impairment (or Likert) scale (being 1 the lowest QoE and 5 the highest QoE, see Table 2), called 5-MOS in this work, and a variant 10-point 
impairment scale (being 1 the lowest QoE and 10 the highest QoE), called 10-MOS in this work. The 10-MOS variant was also considered because it provides higher granularity for evaluating the quality degradation, as well as for discerning any differences between the employed playout adjustment strategies. Other QoE-related works have also used variants of the 5-MOS metric, using 10-point, and even 100-point impairment scales (e.g., [10, 11]).

Table 2. Mean Opinion Score (MOS) Metric

\begin{tabular}{c|c|c}
\hline \hline MOS & Quality & Impairment \\
\hline 5 & Excellent & Imperceptible \\
\hline 4 & Good & Perceptible, but not annoying \\
\hline 3 & Fair & Slightly annoying \\
\hline 2 & Poor & Annoying \\
\hline 1 & Bad & Very annoying \\
\hline
\end{tabular}

In terms of MOS ratings, the results for the two considered video sequences slightly vary. It seems that playout adjustments are slightly more noticeable in fast motion scenes (Video Sequence 2) than in slow motion scenes (Video Sequence 1), both containing natural speech and human gestures, as noticed in [8] and in [10]. However, the obtained results follow an identical tendency and behavior regarding the analyzed test conditions, obtaining the same findings in both sequences, thus corroborating them. Therefore, without loss of generality, we focus the discussion of the obtained results on Video Sequence 1 (the results for Video Sequence 2 are in the supporting document).

Figure 8 shows the mean and the 95\% Confidence Interval of the obtained results for Video Sequence 1, for both 5-MOS (in red, and with 'o' marks) and 10-MOS (in blue, and with ' $\mathrm{x}$ ' marks), grouped according to the asynchrony value and to the type of test condition. The significance of the differences between the 5-point MOS ratings for each test condition was determined by using the Wilcoxon signed rank test, at a significance level of 0.05 . It is a nonparametric statistical hypothesis test commonly used to assess whether two related samples differ [14], and it is typically used as an alternative to the paired Student's t-test when the ratings are discrete and the population cannot be assumed to be normally distributed. For example, it has been used in related works, such as in [15] and [16].

From the results, several findings can be highlighted:

- Playout skips and, especially, pauses are very annoying to users (as reflected in [3] and in [4]). In general, AMP strategies provide better QoE than aggressive playout adjustment techniques.

- Slowing down the playout rate is more critical than fasting it up (as noticed in [10] and in [11]).

- Cubic AMP provides better QoE than Quadratic and Linear AMP, when both slowing down and fasting up the playout rate. Indeed, when considering $\Delta T_{\text {Liner }}$ as the 
adjustment period in all the AMP strategies, Cubic AMP still slightly outperforms Linear AMP, while it does not happen for Quadratic AMP.

- Related to the previous point, having a longer adjustment period when using Cubic AMP compared to when using Linear AMP (50\% longer) does not have a negative impact on the perceived QoE.

- When the playout process is lagged, only the use of Cubic AMP allows correcting the asynchrony (or recovering from a potential buffer underflow situation) without being noticed by users, as there are no significant differences between the Reference and Cubic AMP - Fast Up test conditions $(p=0.1050>0.05$ for Video Sequence 1, and $p=0.1250>0.05$ for Video Sequence 2). This is the unique test condition for which there are no significant differences compared to the Reference test condition.

It can also be appreciated that the tendencies of the obtained results are very similar for the two considered MOS variants (indeed, MOS-5 $\approx($ MOS-10)/2). In order to confirm the similarity of the results when using both MOS variants, a new metric, called MOS degradation, has been defined, with the goal of assessing the degradation of the MOS value for each test condition compared to the one for the Reference test condition (i.e., the one without playout adjustments):

$$
M O S_{\text {_ degradatio }} n=\frac{M O S_{\text {_reference }}-M O S_{\text {_condition }}}{M O S_{\text {reference }}}
$$

The values of the MOS degradation metric for each MOS variant can be seen in Figure 9. It can be appreciated that the results in terms of degradation are very similar for each MOS variant (MOS-5 and MOS-10), except for the aggressive adjustment techniques (i.e., playout skips \& pauses), because the lowest value in both rating scales is " 1 ". This simple test confirms the very similar behavior of the results of the subjective evaluation when using both variants of the MOS metric. 


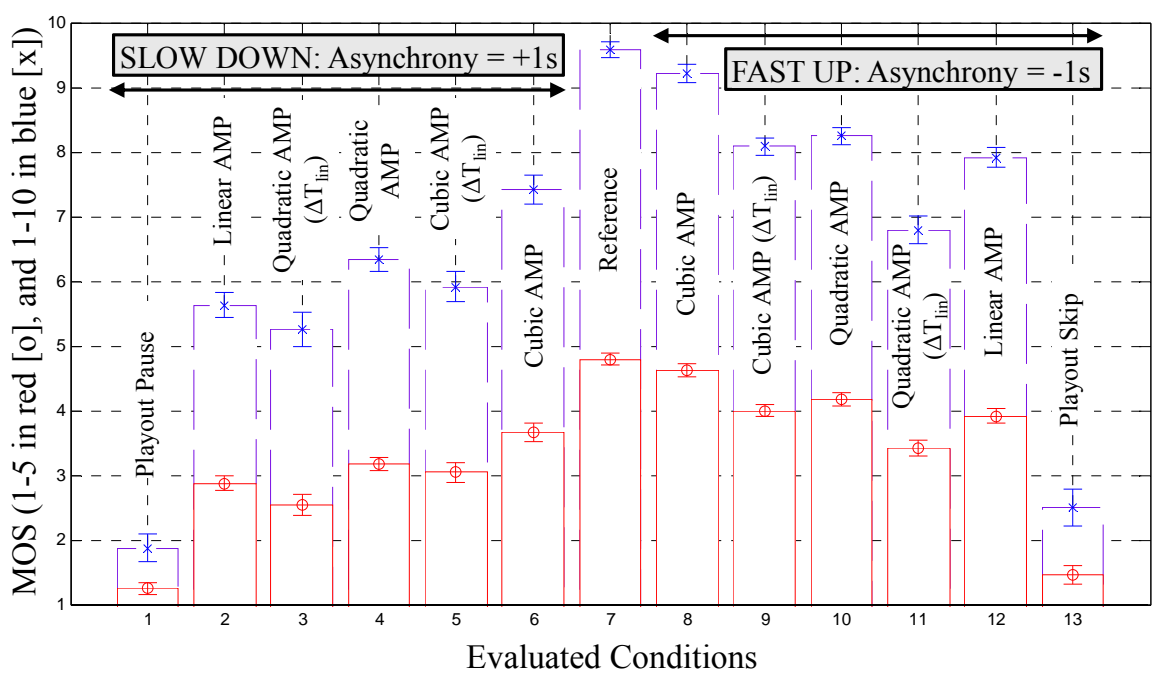

Figure 8. Impact of the different playout adjustment techniques on the QoE for video sequence 1 .

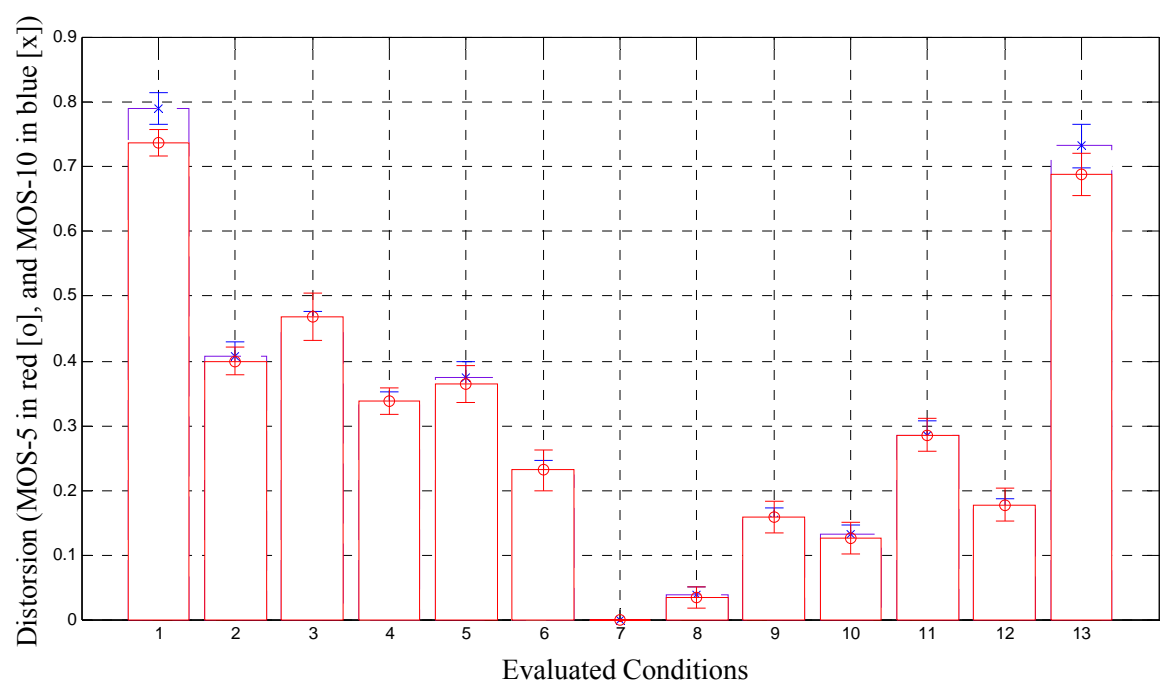

Figure 9. MOS degradation for each MOS variant and for each test condition for video sequence 1 .

\section{Conclusions and Future Work}

In this paper, we have proposed a novel cubic interpolation method for AMP, which we called Cubic AMP. Our Cubic AMP strategy outperforms the other existing Linear and Quadratic AMP strategies (based on adopting linear and quadratic interpolation methods, respectively) in terms of the smoothness of the playout curve (by avoiding bends throughout the playout adjustment process), and significantly reduces the duration of the adjustment period compared to Quadratic AMP (gain of 25\%), without increasing the computational complexity. It has also been proved and discussed that the use of higher-order polynomial interpolation methods does not provide any benefit compared to Cubic AMP. Most interestingly, the results of subjective tests have confirmed that Cubic AMP provides better QoE than Linear and Quadratic AMP. 
Therefore, our research goal has been met and, given the promising performance of Cubic AMP, we believe it is an outstanding contribution and will be relevant in the design of forthcoming AMP solutions and, therefore, multimedia systems. This is because, independently of the goal of the playout adjustments and the criteria for triggering them (Table 1), the best adjustment technique for maximizing the users' perceived QoE must be devised and employed. Therefore, Cubic AMP can be beneficial not only for IDMS, but also for the other purposes reflected in Table 1 (e.g., recovering for buffer underflow/overflow situations, inter-media synchronization...).

Future research will be targeted on determining the allowable thresholds for the playout rate variation $\left(\varphi_{\max }\right)$, for both audio and video (as it has also been noticed that the adjustments of the playout processes are more critical for audio than for video), when both slowing down and fasting up the playout rate. We also plan to investigate the impact of the magnitudes of both the asynchrony thresholds $(\Delta \tau)$ and the duration of the adjustment period $(\Delta T)$ on the QoE. These research tasks will help to determine the benefits of using Cubic AMP for different values of these parameters and conditions, and to confirm that, independently of the magnitude of both the playout adjustment (e.g., the asynchrony that needs to be corrected) and the upper threshold for the playout rate variation, the specific adjustment technique to be performed plays a key role when performing AMP. Finally, we also plan to evaluate the benefits of using Cubic AMP in content-aware systems (as in [8] and in [10]) and, if the results are satisfactory, adopting it in a previously designed eventdriven IDMS-enabled systems [17].

\section{ACKNOWLEDGMENT}

This work has been funded, partially, by the "Fondo Europeo de Desarrollo Regional (FEDER)" and the Spanish Ministry of Economy and Competitiveness, under its R\&D\&I Support Program, in project with Ref. TEC2013-45492-R.

\section{REFERENCES}

[1] M. Montagud, F. Boronat, "On the Use of Adaptive Media Playout for Inter-Destination Synchronization”, IEEE Communications Letters, 15(8), pp. 863-865, August 2011.

[2] M. Montagud, F. Boronat, H. Stokking, P. Cesar, "Design, Development and Assessment of Control Schemes for IDMS in a Standardized RTCP-based Solution", Computer Networks, 70(9), pp.240-259, September 2014.

[3] Y. Ishibashi, S. Tasaka, H. Ogawa, "Media Synchronization Quality of Reactive Control Schemes", IEICE Transactions on Communications, 86 (10), pp.3103-3113, October 2003.

[4] T. Hossfeld, M. Seufert, M. Hirth, T. Zinner, P. Tran-Gia and R. Schatz, "Quantification of YouTube QoE via Crowdsourcing", IEEE International Symposium on Multimedia (IEEE ISM'11), pp. 494-499, Dana Point CA, December, 2011.

[5] M. Li, T. Lin, S. Cheng, "Arrival process-controlled adaptive media playout with multiple thresholds for video streaming”, Multimedia Systems, 18(5), pp. 391-407, October 2012. 
[6] M. Kalman, E. Steinbach, B. Girod, "Adaptive media playout for low-delay video streaming over errorprone channels", IEEE Transactions on Circuits and Systems for Video Technology, 14(6), pp.841-851, June 2004.

[7] H. Chuang, C. Huang, T. Chiang, "Content-Aware Adaptive Media Playout Controls for Wireless Video Streaming”, IEEE Transactions on Multimedia (IEEE TMM), 9(6), pp. 1273-1283, October 2007.

[8] Y. Li, A. Markopoulou, J. Apostolopoulos, N. Bambos, "Content-Aware Playout and Packet Scheduling for Video Streaming Over Wireless Links", IEEE Transactions on Multimedia (IEEE TMM), 10(5), pp.885-895, August 2008.

[9] Y. F. Su, Y. H. Yang, M. T. Lu and H. H. Chen, "Smooth control of adaptive media playout for video streaming”, IEEE Transactions on Multimedia (IEEE TMM), 11(7), pp. 1331-1339, November 2009.

[10]B. Rainer, C. Timmerer, “A subjective evaluation using crowdsourcing of Adaptive Media Playout utilizing audio-visual content features", IEEE Network Operations and Management Symposium (IEEE NOMS 2014), Krakow, pp. 1-7, May 2014.

[11] B. Rainer, C. Timmerer, "A Quality of Experience Model for Adaptive Media Playout", Sixth International Workshop on Quality of Multimedia Experience (QoMEX 2014), pp. 177-182, Singapore, September 2014.

[12]M. Montagud. F. Boronat, H. Stokking, R. van Brandenburg, "Inter-Destination Multimedia Synchronization; Schemes, Use Cases and Standardization”, Multimedia Systems, 18(6), pp. 459-482, November 2012.

[13]ITU-T Recommendation P.910, "Subjective video quality assessment methods for multimedia applications", International Telecommunication Union, Geneva, Switzerland, April 2008.

[14] J.J. Higgins, "Introduction to modern nonparametric statistics", Duxbury Advanced Series, Ed. Thomson-Brooks/Cole, Pacific Grove, CA, 2004.

[15] T. De Pessemier, K. De Moor, W. Joseph, L. De Marez and L. Martens, "Quantifying the Influence of Rebuffering Interruptions on the User's Quality of Experience During Mobile Video Watching”, IEEE Transactions on Broadcasting, 59(1), pp. 47-61, March 2013.

[16] G. Lavoué, M.C. Larabi, L. Vasa, “On the Efficiency of Image Metrics for Evaluating the Visual Quality of 3D Models”, IEEE Transactions on Visualization and Computer Graphics, 2015.

[17] M. Montagud, F. Boronat, H. Stokking, "Early Event-Driven (EED) RTCP Feedback for Rapid IDMS", The 21st ACM International Conference on Multimedia (ACM MM 2013), Barcelona (Spain), October 2013. 


\section{SUPPORTING DOCUMENT}

\section{ANNEX I: Comparison between Cubic and Quartic polynomial interpolation}

The expression for the cubic polynomial interpolation curve for each time $t$ in $\left[t_{0}, t_{1}\right]$ is given by (see Equation 5 in the paper):

$$
\begin{aligned}
& \qquad \begin{array}{l}
\hat{P}(\tau)=-(\theta-\mu)\left(t_{1}+t_{0}\right) \tau^{3}+(\theta-\mu)\left(2 t_{1}+t_{0}\right) \tau^{2}+\mu\left(t_{1}-t_{0}\right) \tau+\mu t_{0} \\
\text { where } \tau=\frac{t-t_{0}}{t_{1}-t_{0}} \in[0,1]
\end{array}
\end{aligned}
$$

The expression for the quartic polynomial interpolation curve for each time $t$ in $\left[t_{0}, t_{1}\right]$ is given by:

$$
\hat{P}(\tau)=(\mu-\theta)\left(t_{0}+2 t_{1}\right) \tau^{4}-(\mu-\theta)\left(t_{0}+3 t_{1}\right) \tau^{3}+\mu\left(t_{1}-t_{0}\right) \tau+\mu t_{0}
$$

Therefore, the computational complexity for calculating the interpolation curve when using a quartic polynomial is not much higher than when using a cubic polynomial.

However, the main difference in terms of computational complexity appears when computing the duration of the adjustment period $(\Delta T)$ for both cubic interpolation $\left(\Delta T_{c u b}\right)$ and quartic interpolation $\left(\Delta T_{\text {quar }}\right)$.

$$
\Delta t_{\text {cub }}=3 \Delta \tau \frac{\left(\varphi_{\max } \mu-2 \varepsilon\right)-\sqrt{\varphi_{\max } \mu\left(\varphi_{\max } \mu-\varepsilon\right)}}{\varepsilon\left(4 \varepsilon-3 \varphi_{\max } \mu\right)}
$$

, being $\varphi_{\max }$ the upper threshold for the playout rate variation (i.e., $\varphi_{\max }=25 \%$ ).

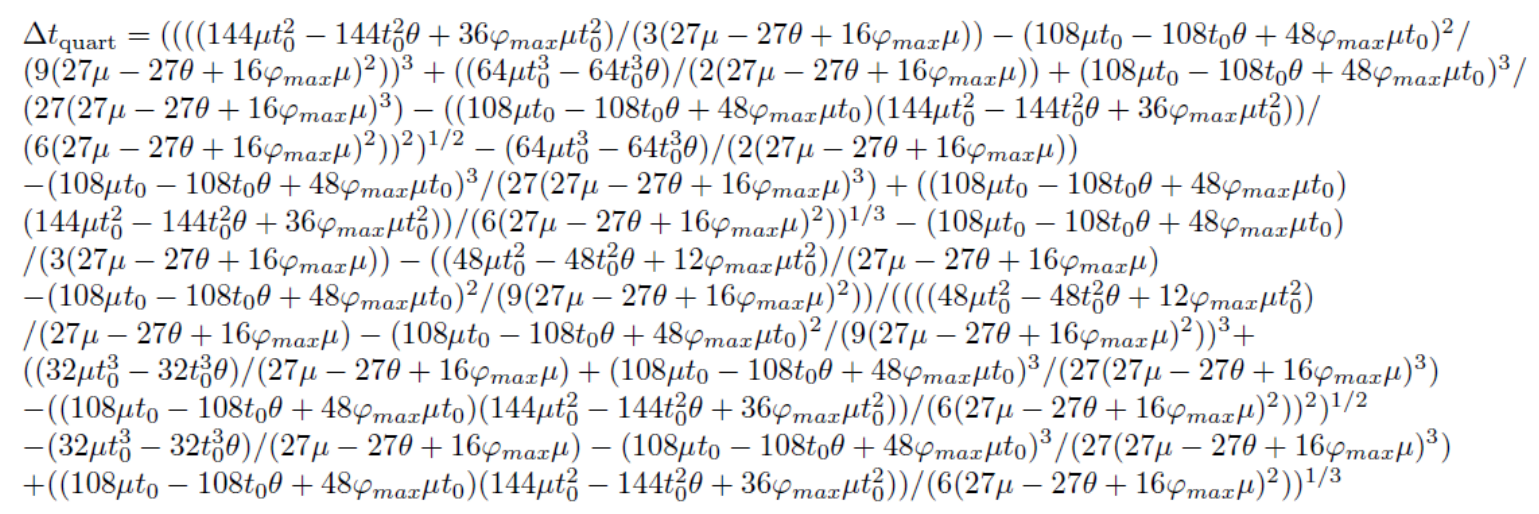

Moreover, the duration of the adjustment period is $30 \%$ longer or more when using a quartic polynomial than when using a cubic polynomial interpolation (see Figure I.1), i.e.:

$$
\Delta T_{\text {quartic }} \approx 1.3 \cdot \Delta T_{\text {cubic }}
$$



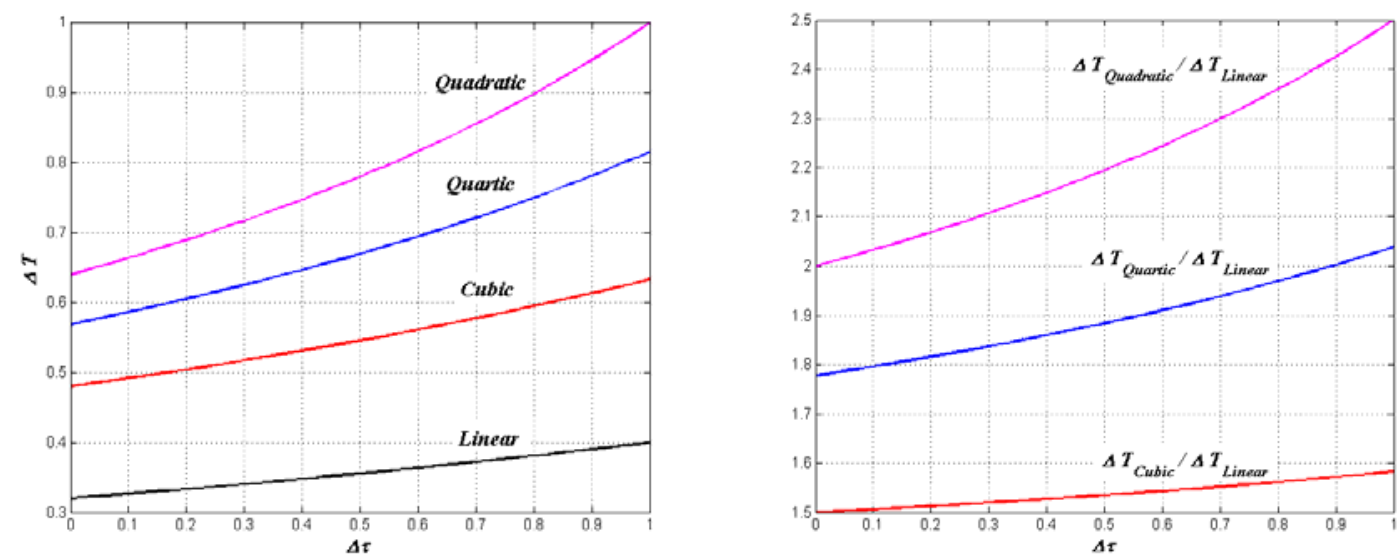

Figure I.1. Comparison of $\Delta T$ versus $\Delta \tau$ for Linear, Quadratic, Cubic and Quartic AMP $\left(\varphi_{\max }=0.25\right)$. 
ANNEX II: Results of the Wilcoxon signed rank tests (significance level of 0.05 )

Table II.1. Reference Condition compared to the rest of conditions in Sequence 1

\begin{tabular}{|c|c|}
\hline & (Hidden) Reference \\
\hline Cubic AMP - Fast Up & $\mathbf{p}=\mathbf{0 . 1 0 5 0}>\mathbf{0 . 0 5}$ (No difference!) \\
\hline Quadratic AMP - Fast Up & $\mathrm{p}=1.2207 \mathrm{e}-04<0.05$ \\
\hline Linear AMP - Fast Up & $\mathrm{p}=6.3178 \mathrm{e}-05<0.05$ \\
\hline Playout Skip & $\mathrm{p}=1.1784 \mathrm{e}-05<0.05$ \\
\hline Cubic AMP - Slow Down & $\mathrm{p}=4.2594 \mathrm{e}-05<0.05$ \\
\hline Quadratic AMP - Slow Down & $\mathrm{p}=8.8871 \mathrm{e}-06<0.05$ \\
\hline Linear AMP - Slow Down & $\mathrm{p}=6.2562 \mathrm{e}-06<0.05$ \\
\hline Playout Pause & $\mathrm{p}=9.9959 \mathrm{e}-06<0.05$ \\
\hline
\end{tabular}

Table II.2. Cubic AMP compared to the other strategies for a lagged playout curve in Sequence 1

\begin{tabular}{|c|c|}
\hline & Cubic AMP - Fast Up \\
\hline Quadratic AMP - Fast Up & $\mathrm{p}=9.7656 \mathrm{e}-04<0.05$ \\
\hline Linear AMP - Fast Up & $\mathrm{p}=9.6166 \mathrm{e}-05<0.05$ \\
\hline Playout Skip & $\mathrm{p}=1.3494 \mathrm{e}-05<0.05$ \\
\hline
\end{tabular}

Table II.3. Cubic AMP compared to the other strategies for an advanced playout curve in Sequence 1

\begin{tabular}{|c|c|}
\hline & Cubic AMP - Slow Down \\
\hline Quadratic AMP - Slow Down & $\mathrm{p}=0.0099<0.05$ \\
\hline Linear AMP - Slow Down & $\mathrm{p}=3.3813 \mathrm{e}-05<0.05$ \\
\hline Playout Pause & $\mathrm{p}=1.2456 \mathrm{e}-05<0.05$ \\
\hline
\end{tabular}

Table II.4. Reference Condition compared to the rest of conditions in Sequence 2

\begin{tabular}{|c|c|}
\hline & (Hidden) Reference \\
\hline Cubic AMP - Fast Up & $\mathbf{p}=\mathbf{0 . 1 2 5 0}>\mathbf{0 . 0 5}$ (No difference!) \\
\hline Quadratic AMP - Fast Up & $\mathrm{p}=4.7777 \mathrm{e}-05<0.05$ \\
\hline Linear AMP - Fast Up & $\mathrm{p}=1.0212 \mathrm{e}-05<0.05$ \\
\hline Playout Skip & $\mathrm{p}=8.8871 \mathrm{e}-06<0.05$ \\
\hline Cubic AMP - Slow Down & $\mathrm{p}=1.5203 \mathrm{e}-05<0.05$ \\
\hline Quadratic AMP - Slow Down & $\mathrm{p}=6.2562 \mathrm{e}-06<0.05$ \\
\hline Linear AMP - Slow Down & $\mathrm{p}=9.2555 \mathrm{e}-06<0.05$ \\
\hline Playout Pause & $\mathrm{p}=1.1033 \mathrm{e}-05<0.05$ \\
\hline
\end{tabular}

Table II.5. Cubic AMP compared to the other strategies for a lagged playout curve in Sequence 2

\begin{tabular}{|c|c|}
\hline & Cubic AMP - Fast Up \\
\hline Quadratic AMP - Fast Up & $\mathrm{p}=2.4414 \mathrm{e}-04<0.05$ \\
\hline Linear AMP - Fast Up & $\mathrm{p}=5.6994 \mathrm{e}-05<0.05$ \\
\hline Playout Skip & $\mathrm{p}=1.0678 \mathrm{e}-05<0.05$ \\
\hline
\end{tabular}

Table II.6. Cubic AMP compared to the other strategies for an advanced playout curve in Sequence 2

\begin{tabular}{|c|c|}
\hline & Cubic AMP - Slow Down \\
\hline Quadratic AMP - Slow Down & $\mathrm{p}=1.2207 \mathrm{e}-04<0.05$ \\
\hline Linear AMP - Slow Down & $\mathrm{p}=7.7442 \mathrm{e}-06<0.05$ \\
\hline Playout Pause & $\mathrm{p}=6.2790 \mathrm{e}-06<0.05$ \\
\hline
\end{tabular}




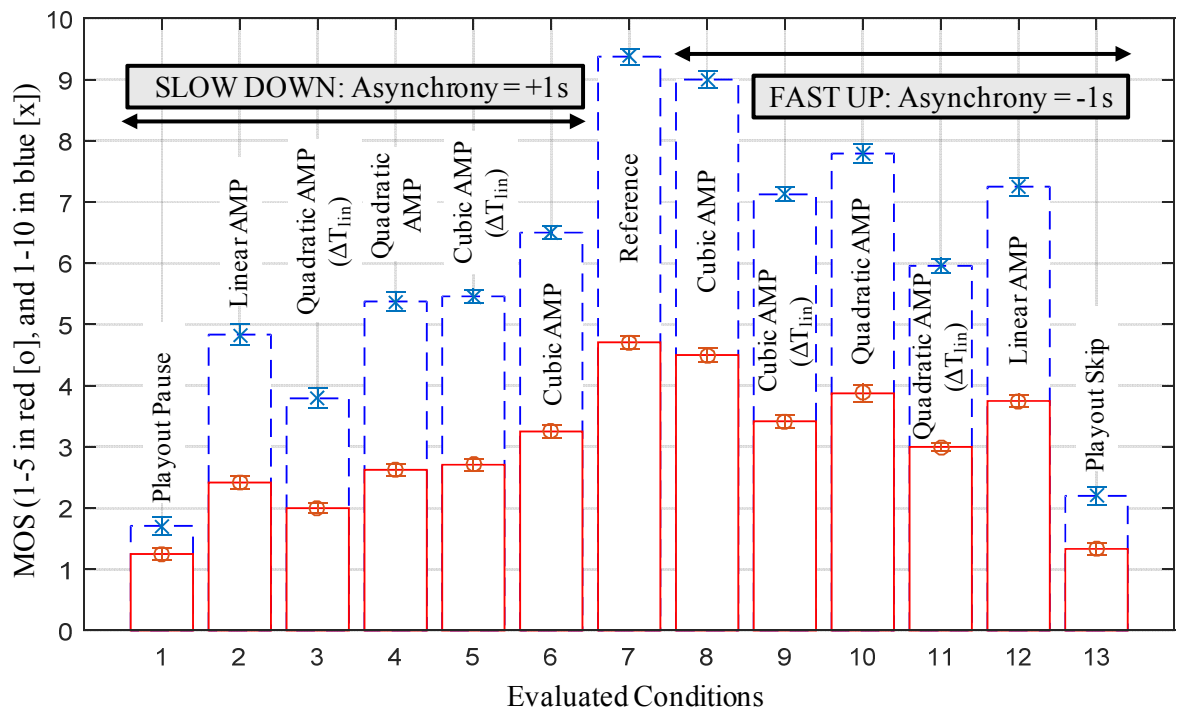

Figure II.1. Impact of the different playout adjustment techniques on the QoE for Video Sequence 2. 